\title{
MAGIC gamma-ray and multi-frequency observations of flat spectrum radio quasar PKS 1510-089 in early 2012
}

J. Aleksić ${ }^{1}$, S. Ansoldi ${ }^{2}$, L. A. Antonelli ${ }^{3}$, P. Antoranz ${ }^{4}$, A. Babic ${ }^{5}$, P. Bangale ${ }^{6}$, U. Barres de Almeida ${ }^{6}$, J. A. Barrio ${ }^{7}$, J. Becerra González $^{8,25}$, W. Bednarek ${ }^{9}$, E. Bernardini ${ }^{10}$, A. Biland ${ }^{11}$, O. Blanch ${ }^{1}$, S. Bonnefoy ${ }^{7}$, G. Bonnoli ${ }^{3}$, F. Borracci ${ }^{6}$, T. Bretz ${ }^{12,26}$, E. Carmona ${ }^{13}$, A. Carosi ${ }^{3}$,

D. Carreto Fidalgo ${ }^{7}$, P. Colin ${ }^{6}$, E. Colombo ${ }^{8}$, J. L. Contreras ${ }^{7}$, J. Cortina ${ }^{1}$, S. Covino ${ }^{3}$, P. Da Vela ${ }^{4}$, F. Dazzi ${ }^{6}$, A. De Angelis ${ }^{2}$, G. De Caneva ${ }^{10, \star}$, B. De Lotto ${ }^{2}$, C. Delgado Mendez ${ }^{13}$, M. Doert ${ }^{14}$, A. Domínguez ${ }^{15,27}$, D. Dominis Prester ${ }^{5}$, D. Dorner ${ }^{12}$, M. Doro $^{16}$, S. Einecke $^{14}$, D. Eisenacher ${ }^{12}$, D. Elsaesser ${ }^{12}$, E. Farina ${ }^{17}$, D. Ferenc ${ }^{5}$, M. V. Fonseca ${ }^{7}$, L. Font ${ }^{18}$, K. Frantzen ${ }^{14}$, C. Fruck ${ }^{6}$, R. J. García López ${ }^{8}$, M. Garczarczyk ${ }^{10}$,

D. Garrido Terrats ${ }^{18}$, M. Gaug ${ }^{18}$, N. Godinović ${ }^{5}$, A. González Muñoz ${ }^{1}$, S. R. Gozzini ${ }^{10}$, D. Hadasch ${ }^{19}$, M. Hayashida ${ }^{20}$, J. Herrera ${ }^{8}$, A. Herrero $^{8}$, D. Hildebrand ${ }^{11}$, J. Hose ${ }^{6}$, D. Hrupec ${ }^{5}$, W. Idec ${ }^{9}$, V. Kadenius ${ }^{21}$, H. Kellermann ${ }^{6}$, K. Kodani ${ }^{20}$, Y. Konno ${ }^{20}$, J. Krause ${ }^{6}$, H. Kubo ${ }^{20}$, J. Kushida ${ }^{20}$, A. La Barbera ${ }^{3}$, D. Lelas ${ }^{5}$, N. Lewandowska ${ }^{12}$, E. Lindfors ${ }^{21,28, \star}$, S. Lombardi ${ }^{3}$, M. López ${ }^{7}$, R. López-Coto ${ }^{1}$, A. López-Oramas ${ }^{1}$, E. Lorenz ${ }^{6}$, I. Lozano $^{7}$, M. Makariev ${ }^{22}$, K. Mallot ${ }^{10}$, G. Maneva ${ }^{22}$, N. Mankuzhiyil ${ }^{2}$, K. Mannheim ${ }^{12}$, L. Maraschi ${ }^{3}$, B. Marcote ${ }^{23}$, M. Mariotti $^{16}$, M. Martínez ${ }^{1}$, D. Mazin ${ }^{6}$, U. Menzel ${ }^{6}$, M. Meucci ${ }^{4}$, J. M. Miranda ${ }^{4}$, R. Mirzoyan ${ }^{6}$, A. Moralejo ${ }^{1}$, P. Munar-Adrover ${ }^{23}$, D. Nakajima ${ }^{20}$, A. Niedzwiecki ${ }^{9}$, K. Nilsson ${ }^{21,28}$, K. Nishijima ${ }^{20}$, K. Noda ${ }^{6}$, N. Nowak ${ }^{6}$, R. Orito ${ }^{20}$, A. Overkemping ${ }^{14}$, S. Paiano ${ }^{16}$, M. Palatiello ${ }^{2}$, D. Paneque ${ }^{6}$, R. Paoletti ${ }^{4}$, J. M. Paredes ${ }^{23}$, X. Paredes-Fortuny ${ }^{23}$, S. Partini ${ }^{4}$, M. Persic ${ }^{2,29}$, F. Prada ${ }^{15,30}$, P. G. Prada Moroni ${ }^{24}$, E. Prandini ${ }^{11}$, S. Preziuso ${ }^{4}$, I. Puljak ${ }^{5}$, R. Reinthal ${ }^{21}$, W. Rhode ${ }^{14}$, M. Ribó ${ }^{23}$, J. Rico ${ }^{1}$, J. Rodriguez Garcia ${ }^{6}$, S. Rügamer ${ }^{12}$, A. Saggion ${ }^{16}$, T. Saito ${ }^{20, \star}$, K. Saito $^{20, \star}$,

K. Satalecka ${ }^{7}$, V. Scalzotto ${ }^{16}$, V. Scapin ${ }^{7}$, C. Schultz ${ }^{16}$, T. Schweizer ${ }^{6}$, S. N. Shore ${ }^{24}$, A. Sillanpää ${ }^{21}$, J. Sitarek ${ }^{1, \star}$, I. Snidaric ${ }^{5}$, D. Sobczynska ${ }^{9}$, F. Spanier ${ }^{12}$, V. Stamatescu ${ }^{1}$, A. Stamerra ${ }^{3}$, T. Steinbring ${ }^{12}$, J. Storz ${ }^{12}$, M. Strzys ${ }^{6}$, S. Sun ${ }^{6}$, T. Surić ${ }^{5}$, L. Takalo ${ }^{21}$, H. Takami $^{20}$, F. Tavecchio ${ }^{3, \star}$, P. Temnikov ${ }^{22}$, T. Terzić ${ }^{5}$, D. Tescaro ${ }^{8}$, M. Teshima ${ }^{6}$, J. Thaele ${ }^{14}$, O. Tibolla ${ }^{12}$, D. F. Torres ${ }^{19}$, T. Toyama ${ }^{6}$, A. Treves ${ }^{17}$, M. Uellenbeck $^{14}$,

P. Vogler ${ }^{11}$, R. M. Wagner ${ }^{6,31}$, F. Zandanel ${ }^{15,32}$, R. Zanin ${ }^{23}$ (the MAGIC Collaboration), F. Lucarelli ${ }^{34}$, C. Pittori ${ }^{34}$, S. Vercellone ${ }^{35}$,

F. Verrecchia ${ }^{34}$ (for the AGILE Collaboration), S. Buson ${ }^{16}$, F. D’Ammando ${ }^{33,44}$, L. Stawarz ${ }^{62,63}$, M. Giroletti ${ }^{44}$, M. Orienti ${ }^{44}$, (for the Fermi-LAT Collaboration), C. Mundell ${ }^{36}$, I. Steele ${ }^{36}$, B. Zarpudin ${ }^{37}$, C. M. Raiteri ${ }^{38}$, M. Villata ${ }^{38}$, A. Sandrinelli ${ }^{16}$, A. Lähteenmäki ${ }^{39,40}$,

J. Tammi ${ }^{39}$, M. Tornikoski ${ }^{39}$, T. Hovatta ${ }^{41}$, A. C. S. Readhead ${ }^{41}$, W. Max-Moerbeck ${ }^{41}$, J. L. Richards ${ }^{42}$, S. Jorstad ${ }^{43}$, A. Marscher ${ }^{43}$,

M. A. Gurwell ${ }^{45}$, V. M. Larionov ${ }^{46,47,48}$, D. A. Blinov ${ }^{49,46}$, T. S. Konstantinova ${ }^{46}$, E. N. Kopatskaya ${ }^{46}$, L. V. Larionova ${ }^{46}$, E. G. Larionova $^{46}$, D. A. Morozova ${ }^{46}$, I. S. Troitsky ${ }^{46}$, A. A. Mokrushina ${ }^{46}$, Yu. V. Pavlova ${ }^{46}$, W. P. Chen ${ }^{50}$, H. C. Lin $^{50}$, N. Panwar ${ }^{50}$ I. Agudo $^{51,52,43}$ C. Casadio $^{51}$, J. L. Gómez ${ }^{51}$, S. N. Molina ${ }^{51}$, O. M. Kurtanidze ${ }^{53,54,55}$, M. G. Nikolashvili ${ }^{53}$, S. O. Kurtanidze ${ }^{53}$, R. A. Chigladze ${ }^{53}$, J. A. Acosta-Pulido ${ }^{56,57}$, M. I. Carnerero ${ }^{38,56,57}$, A. Manilla-Robles ${ }^{57}$, E. Ovcharov ${ }^{58}$, V. Bozhilov ${ }^{58}$, I. Metodieva ${ }^{58}$, M. F. Aller ${ }^{59}$, H. D. Aller ${ }^{59}$, L. Fuhrman ${ }^{60}$, E. Angelakis ${ }^{60}$, I. Nestoras ${ }^{60}$, T. P. Krichbaum ${ }^{60}$, J. A. Zensus ${ }^{60}$, H. Ungerechts ${ }^{61}$, and A. Sievers ${ }^{61}$

(Affiliations can be found after the references)

Received 22 January 2014 / Accepted 30 May 2014

\section{ABSTRACT}

Aims. Amongst more than fifty blazars detected in very high energy (VHE, $E>100 \mathrm{GeV}$ ) $\gamma$ rays, only three belong to the subclass of flat spectrum radio quasars (FSRQs). The detection of FSRQs in the VHE range is challenging, mainly because of their soft spectra in the GeV-TeV regime. MAGIC observed PKS 1510-089 $(z=0.36)$ starting 2012 February 3 until April 3 during a high activity state in the high energy (HE, $E>100 \mathrm{MeV}) \gamma$-ray band observed by AGILE and Fermi. MAGIC observations result in the detection of a source with significance of 6.0 standard deviations $(\sigma)$. We study the multi-frequency behaviour of the source at the epoch of MAGIC observation, collecting quasi-simultaneous data at radio and optical (GASP-WEBT and F-Gamma collaborations, REM, Steward, Perkins, Liverpool, OVRO, and VLBA telescopes), X-ray (Swift satellite), and HE $\gamma$-ray frequencies.

Methods. We study the VHE $\gamma$-ray emission, together with the multi-frequency light curves, $43 \mathrm{GHz}$ radio maps, and spectral energy distribution (SED) of the source. The quasi-simultaneous multi-frequency SED from the millimetre radio band to VHE $\gamma$ rays is modelled with a one-zone inverse Compton model. We study two different origins of the seed photons for the inverse Compton scattering, namely the infrared torus and a slow sheath surrounding the jet around the Very Long Baseline Array (VLBA) core.

Results. We find that the VHE $\gamma$-ray emission detected from PKS 1510-089 in 2012 February-April agrees with the previous VHE observations of the source from 2009 March-April. We find no statistically significant variability during the MAGIC observations on daily, weekly, or monthly time scales, while the other two known VHE FSRQs (3C 279 and PKS 1222+216) have shown daily scale to sub-hour variability. The $\gamma$-ray SED combining AGILE, Fermi and MAGIC data joins smoothly and shows no hint of a break. The multi-frequency light curves suggest a common origin for the millimetre radio and HE $\gamma$-ray emission, and the HE $\gamma$-ray flaring starts when the new component is ejected from the 43 GHz VLBA core and the studied SED models fit the data well. However, the fast HE $\gamma$-ray variability requires that within the modelled large emitting region, more compact regions must exist. We suggest that these observed signatures would be most naturally explained by a turbulent plasma flowing at a relativistic speed down the jet and crossing a standing conical shock.

Key words. galaxies: active - galaxies: jets - gamma rays: galaxies - quasars: individual: PKS 1510-089

^ Corresponding authors: E. Lindfors, e-mail: elilin@utu.fi; F. Tavecchio, e-mail: fabrizio.tavecchio@brera.inaf.it; K. Saito, e-mail: ksaito@icrr.utokyo.ac.jp; J. Sitarek, e-mail: jsitarek@ifae.es; G. De Caneva, e-mail: gessica.de.caneva@desy.de 


\section{Introduction}

The most numerous extragalactic very high energy (VHE, $E>$ $100 \mathrm{GeV}) \gamma$-ray sources are blazars, which are active galactic nuclei (AGN) with relativistic jets pointing close to our line of sight. Within the blazar group the most numerous VHE $\gamma$-ray emitters are X-ray bright BL Lacertae objects (BL Lacs) while only three blazars of the flat spectrum radio quasars (FSRQs) type have been detected to emit VHE $\gamma$ rays.

Blazars typically show variable emission in all wavebands from radio to $\gamma$ rays. FSRQs are more luminous than BL Lacs at $\gamma$ rays and so they could, in principle, be observed at greater distances at very high energies. The spectral energy distributions (SEDs) of both types of sources show two peaks: the first is generally attributed to synchrotron emission and the second one to inverse Compton (IC) scattering, though hadronic mechanisms have also been proposed for producing the second peak (see e.g. Böttcher et al. 2009). In FSRQs the first peak is usually in the infrared regime, while for BL Lacs it is between infrared and hard X-rays. The optical spectra of FSRQs show broad emission lines, indicative of high velocity gas in the so-called broad line region (BLR) close (0.1 to 1 parsec) to the central engine (e.g. Kaspi et al. 2000), while BL Lacs show very weak or no emission lines in their spectra. Because of these properties FSRQs were not thought to be good candidates to emit VHE $\gamma$ rays: the low synchrotron peak frequency may imply efficient synchrotron cooling, which makes it difficult to produce VHE $\gamma$-ray emission. Additionally, if the $\gamma$ rays are produced close to the central engine, the BLR clouds absorb the $\gamma$-ray emission via pair production. The high redshift also implies strong absorption of VHE $\gamma$ rays by the extragalactic background light (EBL; Stecker et al. 1992; Hauser \& Dwek 2001). Despite these difficulties, MAGIC detected VHE $\gamma$ rays from the FSRQ 3C 279 $(z=0.536)$ in 2006 (Albert et al. 2008a). This discovery was followed by a second detection in 2007 (Aleksić et al. 2011a) and the detection of two other FSRQs PKS $1510-089(z=0.36)$ by H.E.S.S. (Abramowski et al. 2013) in 2009 and PKS 1222+216 $(z=0.432)$ in 2010 (Aleksić et al. 2011b). In this paper we report the detection of VHE $\gamma$ rays from PKS 1510-089 in 2012 February-April (Cortina 2012) by the MAGIC telescopes.

The standard picture for FSRQs is that the $\gamma$ rays are emitted close to the central black hole (so called "near-dissipation zone"), where the external photons from BLR can serve as seed photons for IC scattering (e.g. Hartman et al. 2000). This picture was already challenged in the EGRET era by the observations of a connection between radio outburst and $\gamma$-ray flares (e.g. Jorstad et al. 2001; Lähteenmäki \& Valtaoja 2003; Lindfors et al. 2006). The observations of VHE $\gamma$ rays from FSRQs have further challenged the "near-dissipation zone" emission scenario (see e.g. Aleksić et al. 2011a,b), because in order to produce the observed VHE $\gamma$-ray flux, the MeV $\gamma$-ray flux would have to be much higher than observed. Moreover, the combined HE to VHE $\gamma$-ray spectrum does not show a break at a few tens of $\mathrm{GeV}$ as would be expected if the $\gamma$ rays originated in inside the BLR (e.g. Tavecchio \& Mazin 2009). In addition, at least in some cases (3C 279 in 2007 and PKS 1222+216 in 2010), the VHE $\gamma$-ray detections were coincident with zero-separation epochs of new knots emerging from the $43 \mathrm{GHz}$ Very Long Baseline Array (VLBA) core (Larionov et al. 2008; Jorstad et al. 2012; Marscher et al. 2012), suggesting that VHE $\gamma$ rays could be emitted in these knots, tens of parsecs away from the central engine. Arguments for and against the "near-dissipation zone" are systematically discussed in e.g. Sikora et al. (2009). In general, the main argument against emission originating far away from the central engine has been the fast variability observed in $\gamma$ rays. However, the recent model by Marscher (2014) where relativistic turbulent plasma crosses a standing shock, could potentially explain both the observed radio-gamma connection and the fast variability of $\gamma$ rays.

PKS $1510-089$ is a $\gamma$-ray bright quasar, whose jet exhibits one of the fastest apparent motions (up to $45 c$ ) amongst all blazars (Jorstad et al. 2005). It was discovered in HE $\gamma$ rays by EGRET, but no variability was detected (Hartman et al. 1999), while in the AGILE and Fermi era it has shown several flaring epochs. A variability study of this source with AGILE data in the period 2007 July-2009 October was presented in Verrecchia et al. (2013). The source showed bright flares at radio, optical, $\mathrm{X}$-ray and HE $\gamma$-ray energies at the beginning of 2009 (Marscher et al. 2010; Abdo et al. 2010a; D'Ammando et al. 2011). The discovery of VHE $\gamma$ rays from PKS 1510-089 also took place in this period, displaying a rather low flux $(F(>150 \mathrm{GeV})=(1.0 \pm$ $\left.0.2_{\text {stat }} \pm 0.2_{\text {sys }}\right) \times 10^{-11} \mathrm{ph} \mathrm{cm}^{-2} \mathrm{~s}^{-1}, \sim 3 \%$ of Crab Nebula flux $)$ and a very soft spectrum (with photon index, $\Gamma=5.4 \pm 0.7_{\text {stat }} \pm 0.3_{\text {sys }}$, Abramowski et al. 2013). In HE $\gamma$ rays this outburst consisted of several flares. In X-rays flaring was moderate and not correlated with the $\gamma$-ray flaring, but the last $\gamma$-ray flare was accompanied by a large optical outburst (reaching a peak flux of $18 \mathrm{mJy}$ in the $R$-band while the quiescent level flux is typically $\sim 2 \mathrm{mJy}$ ) and a large radio outburst (reaching a maximum of $4 \mathrm{Jy}, 1-2 \mathrm{Jy}$ being the normal quiescent state flux at $37 \mathrm{GHz}$ ). During the $\gamma$-ray flares the optical electric vector position angle (EVPA) rotated by $>720^{\circ}$ and during the major optical flare, the optical polarisation degree increased to $>30 \%$. In the $43 \mathrm{GHz}$ VLBA maps a superluminal knot emerged from the VLBA core with a zeroseparation epoch essentially simultaneous with this sharp optical flare. Marscher et al. (2010) concluded that the $\gamma$-ray flaring activity was taking place in a knot seen in the VLBA images at later times, placing the emission region distant from the central engine. This and the variable synchrotron to $\gamma$-ray ratio require that there are local sources of seed photons for IC scattering within or just outside the jet (e.g. a slow sheath of a jet). In contrast, based on the ratio between optical and $\gamma$-ray variability Abdo et al. (2010a) concluded that the $\gamma$-ray emission favors an external Compton model where the seed photons are provided by the BLR clouds.

In second half of 2011 the source again showed activity in several bands. First, in 2011 July, there were optical and HE $\gamma$-ray flares accompanied by the rotation of the EVPA by $>380^{\circ}$ (Orienti et al. 2013). In second half of 2011 PKS 1510-089 underwent the brightest radio flare ever observed from the source and there was associated high activity in the HE $\gamma$-ray band. The flare was accompanied by the appearance of a new component in the VLBA jet at $15 \mathrm{GHz}$ (Orienti et al. 2013) and by extremely fast $\gamma$-ray variability with time scales down to $20 \mathrm{~min}$ (e.g. Saito et al. 2013; Foschini et al. 2013). Unfortunately, during this period the source was not observable for ground based optical and $\gamma$-ray instruments.

In 2012 February PKS 1510-089 showed again high activity in HE $\gamma$ rays (Lucarelli et al. 2012). This triggered observations of the source with the MAGIC telescopes which resulted with a significant detection of VHE $\gamma$ rays (Cortina 2012; De Caneva et al. 2012). The results from the MAGIC observations (Sect. 2) are presented together with HE $\gamma$-ray data from AGILE and Fermi (Sect. 3), X-ray data from Swift (Sect. 4), near infrared, optical, ultraviolet (Sect. 5), and radio observations (Sect. 6) from several instruments. A subset of the data presented here have been previously presented in Lindfors et al. (2013), while in this paper we present the full analysis of the multi-frequency 
behaviour of the source during 2012 February-April and compare it with the previous flaring epochs of PKS 1510-089.

\section{MAGIC VHE $\gamma$-ray observations, data analysis, and results}

\subsection{Observations and data analysis}

MAGIC is a system of two $17 \mathrm{~m}$ diameter Imaging Air Cherenkov Telescopes (IACTs) located at the Roque de los Muchachos Observatory on La Palma, one of the Canary Islands $\left(28^{\circ} 46^{\prime} \mathrm{N}, 17^{\circ} 53.4^{\prime} \mathrm{W}\right.$ at $2231 \mathrm{~m}$ a.s.l.). The large collection area of the telescopes and the advanced observational techniques enables us to reach a low energy threshold of $50 \mathrm{GeV}$ (in a normal stereo trigger mode) at low zenith angles. In late 2011 the telescope readout system was upgraded and replaced (Sitarek et al. 2013).

The MAGIC target of opportunity (ToO) observations of PKS 1510-089 were carried out from 2012 February 3 to April 3 (MJD 55 960-56020). During 28 nights $25 \mathrm{~h}$ of data were taken with the stereo trigger, of which $21.4 \mathrm{~h}$ data passed quality selection. The data were collected at zenith angles between $37^{\circ}$ and $49^{\circ}$. The telescopes were operated with the false source tracking method (Fomin et al. 1994), the so-called wobble mode, in which the pointing direction counter-changes every $20 \mathrm{~min}$ between four sky positions at $0.4^{\circ}$ offset with respect to the source position. Four wobble positions improve the background statistics, since three OFF positions can be sampled which reduces the impact of inhomogeneities in the camera acceptance.

We analysed the data in the MARS analysis framework (Moralejo et al. 2009). The images were processed using a cleaning algorithm that accounts for timing information (Aliu et al. 2009). The criteria for core and boundary pixels are eight and four photo-electrons, respectively. These are different from those used for the standard analyses done before the upgrade of the readout (the details are described in Aleksić et al. 2012) mainly due to the different noise level of the new readout system. The random forest (RF) method was used for the gamma-hadron separation (Albert et al. 2008b) using both mono and stereoscopic parameters. The reconstructed shower arrival direction of each telescope was calculated with the RF DISP method (Aleksic et al. 2010), and the weighted mean of the closest pair amongst the reconstructed DISP positions is regarded as the final reconstructed position.

\subsection{Results}

The distributions of squared angular distances between the reconstructed source position and the nominal source position in the camera, the so-called $\theta^{2}$ plot, is shown in Fig.1. The number of the background events was extracted from the three OFF regions which were symmetrical relative to the pointing position. Above the normalised background events, an excess of $539 \gamma$ rays was found. The significance of a signal detection was evaluated following Eq. (17) of Li \& Ma (1983). We found a corresponding significance of $6 \sigma$ from the $21.4 \mathrm{~h}$ observational data. The observation at high zenith angle had a somewhat higher energy threshold of $120 \mathrm{GeV}$, determined from the Monte Carlo rate with an assumed photon index of 4.0.

To derive the energy spectrum of PKS 1510-089 the unfolding procedure (Albert et al. 2007) was performed to correct for a distortion introduced by the detector which has a finite resolution and biases. Moreover, absorption by $\mathrm{e}^{+} \mathrm{e}^{-}$pair creation due to the interaction with the EBL photons was also corrected through the

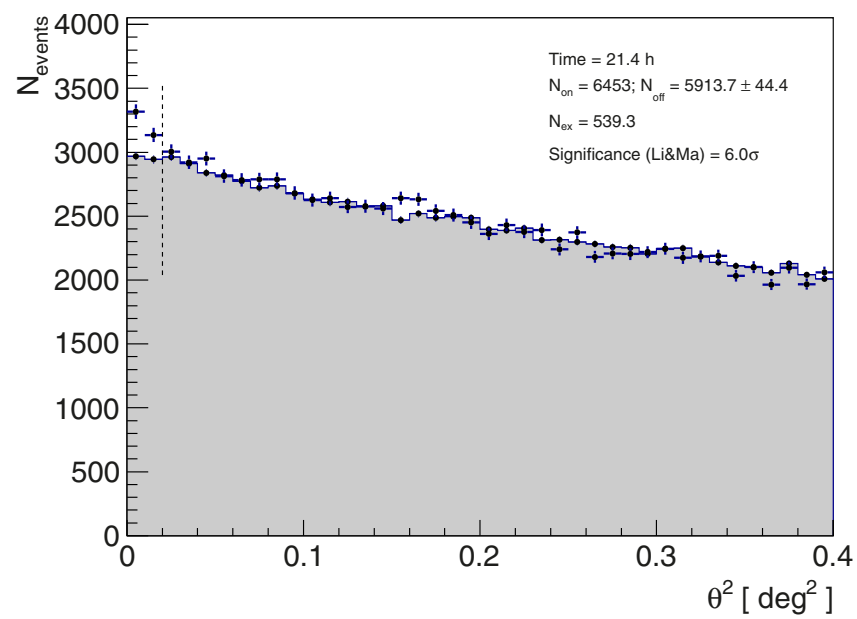

Fig. 1. Distribution of the square of reconstructed shower direction $\left(\theta^{2}\right)$ with respect to the position of PKS 1510-089 for the ON (black points) and the OFF (grey shaded area) in the camera coordinates. The events inside the vertical dashed line, corresponding to the a priori-defined signal region, are used to compute the detection significance.

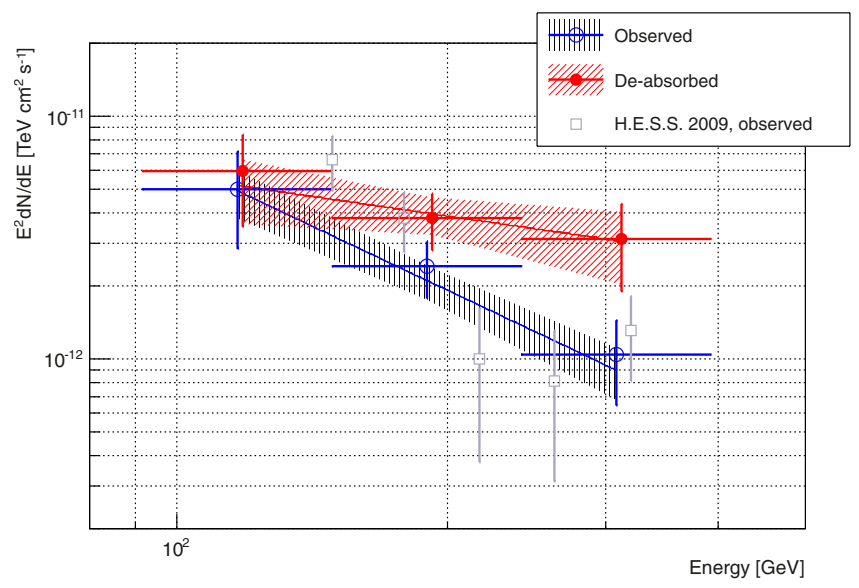

Fig. 2. VHE differential energy spectra of PKS 1510-089 measured by MAGIC in the period between 2012 February 3 and April 3. The blue open circles and the blue shaded region show the observed spectrum and its statistical uncertainty, the red dots and the red shaded region show the de-absorbed spectrum (see text). The grey open squares are the source spectrum observed in March-April 2009 by H.E.S.S. (Abramowski et al. 2013).

same unfolding process, using one of the several state-of-the-art EBL model (Dominguez et al. 2011).

We found that different unfolding methods gave consistent results, and the energy spectrum before the EBL correction can be well reproduced by a power law

$\frac{\mathrm{d} F}{\mathrm{~d} E}=F_{0}\left(\frac{E}{200 \mathrm{GeV}}\right)^{-\Gamma}$,

where $F_{0}=\left(4.8 \pm 0.9_{\text {stat }} \pm 1.3_{\text {sys }}\right) \times 10^{-11} \mathrm{~cm}^{-2} \mathrm{~s}^{-1} \mathrm{TeV}^{-1}$ and $\Gamma=3.8 \pm 0.4_{\text {stat }} \pm 0.3_{\text {sys }}$ are the flux constant at $200 \mathrm{GeV}$ and the photon index, respectively. As PKS 1510-089 is a very weak, steep spectrum VHE source the systematic errors are larger than the ones evaluated in Aleksić et al. (2012). The systematic error in the energy scale is $17 \%$ as in Aleksić et al. (2012). Figure 2 shows the differential energy spectra of PKS 1510-089 measured by MAGIC in 2012. The fitted function and its one sigma error range displayed as the shaded regions were obtained through the forward unfolding, and the 
spectral points were derived using the Bertero unfolding method (Bertero 1989). The spectrum extends up to $\sim 400 \mathrm{GeV}$. The integral flux above $120 \mathrm{GeV}$ was estimated to be $4 \%$ of the Crab Nebula's flux. After the correction for the EBL attenuation the spectrum is still well fitted by a power law with an intrinsic photon index of $\Gamma_{\text {int }}=2.5 \pm 0.6_{\text {stat }}$. The flux and spectrum are in agreement with those observed by H.E.S.S. in March-April 2009 (Abramowski et al. 2013).

The $\gamma$-ray flux variability above $200 \mathrm{GeV}$ was studied on both daily and weekly time scales. The mean flux above $200 \mathrm{GeV}$ of PKS $1510-089$ in this period was $F(>200 \mathrm{GeV})=$ $(3.6 \pm 0.9) \times 10^{-12} \mathrm{ph} \mathrm{cm}^{-2} \mathrm{~s}^{-1}$. The reduced $\chi^{2}$ of the fit with a constant flux is $\chi^{2} / n_{\text {d.o.f. }}=40.5 / 24(2.3 \sigma)$ for daily and $\chi^{2} / n_{\text {d.o.f. }}=7.7 / 4(1.6 \sigma)$ for weekly light curve, consistent with no statistically significant variability. Following the method used in Aleksić et al. (2013) we also estimated how much variability could be hidden in the data. We derived a $3 \sigma$ confidence level upper limit for individual nights/weeks and compared it to the observed mean flux adopting the night-to-night systematic error of $12 \%$ (Aleksić et al. 2012). We found that variability of a factor of eight in nightly scale and factor of 2.5 in the weekly scale could be missed. The weekly light curve is displayed and discussed with the multi-frequency data in Sect. 7. The observed VHE $\gamma$-ray emission, showing only marginal variability over several weeks, displays a different behaviour than other FSRQs (Albert et al. 2008a; Aleksić et al. 2011b,a), but is in agreement with previous observations of PKS 1510-089 by H.E.S.S. in 2009 March-April (Abramowski et al. 2013).

\section{HE $\gamma$-ray observations, data analysis, and results}

We investigate the emission in the $\mathrm{HE} \gamma$-ray range making use of two instruments: AGILE-GRID and Fermi-LAT. The comparison and combination of the HE and VHE $\gamma$-ray results are presented in Sect. 3.3.

\subsection{AGILE}

Astrorivelatore Gamma ad Immagini LEggero (AGILE, Tavani et al. 2009) is a scientific mission devoted to the observation of astrophysical sources of HE $\gamma$ rays in the $30 \mathrm{MeV}-30 \mathrm{GeV}$ energy range, with simultaneous $\mathrm{X}$-ray imaging capability in the $18-60 \mathrm{keV}$ band. The AGILE payload combines for the first time two coaxial detectors: the gamma-ray imaging detector (GRID, composed of a 12-plane silicon-tungsten tracker, a cesium-iodide mini-calorimeter and an anti-coincidence shield) and the hard X-ray detector Super-AGILE. The $\gamma$-ray GRID imager provides good performance in a relatively small and compact instrument due to the use of silicon technology: an effective area of the order of $500 \mathrm{~cm}^{2}$ at several hundred $\mathrm{MeV}$, an angular resolution of around $3.5^{\circ}$ at $100 \mathrm{MeV}$, decreasing below $1^{\circ}$ above $1 \mathrm{GeV}$, a very large field of view $(\sim 2.5 \mathrm{sr})$, as well as accurate timing, positional and altitude information.

During the first $\sim 2.5$ years (2007 July-2009 October), AGILE was operated in "pointing observing mode", characterised by long observations called observation blocks (OBs), typically of two to four weeks duration. Since 2009 November 4 , following a malfunction of the rotation wheel, AGILE is operating in "spinning observing mode", surveying a large fraction (about $70 \%$ ) of the sky each day. Thanks to its sky monitoring capability and fast ground segment alert system distributed amongst the AGILE Data Centre (ADC) and the AGILE team institutes, AGILE is very effective in detecting bright $\gamma$-ray flares from blazars.
Data were analysed applying the AGILE maximum likelihood (ML) analysis on the PKS 1510-089 sky position, using the standard level-3 AGILE-GRID archive at ADC. This archive is composed by counts, exposure and diffuse $\gamma$-ray background (Giuliani et al. 2004) maps generated on several time scales (one day, one week, 28 days) from the official level-2 data archives, publicly available at the ADC site ${ }^{1}$. Maps were generated for $E>100 \mathrm{MeV}$ including all events collected up to $60^{\circ}$ off-axis, excluding south Atlantic anomaly data, and by excluding regions within $10^{\circ}$ from the Earth limb to reduce albedo contamination. The data have been processed with the latest available software and calibrations ${ }^{2}$. For a general description of the AGILE data reduction and of the standard analysis pipeline see Pittori et al. (2009), Vercellone et al. (2010). Systematic errors of the AGILE ML analysis have been estimated to be $\sim 10 \%$ of the flux values (Bulgarelli et al. 2012).

At the beginning of 2012, AGILE detected the PKS 1510-089 in a high state in two distinct periods: one at the end of January-beginning of February, and the other at the end of February-beginning of March. The AGILE-GRID $(E>100 \mathrm{MeV})$ light curves covering the MAGIC observation of PKS 1510-089 from January to March (MJD 55 950-56 000), with two days time binning are shown together with the multifrequency light curves in Sect. 7. In comparing the AGILE and Fermi light curves it should be taken into account that over short time intervals, AGILE might not spectrally resolve the blazar due to low statistics, and in such cases a "standard" fixed spectral photon index value of 2.1 is adopted for the ML analysis. This effect may result in an additional systematic error on the flux (not shown in the figure). By using, for example, a fixed spectral index value of 2.4, AGILE flux values would change on average by a factor $+15 \%$.

The first high state (flare-I) triggered the AGILE alert system and four day quick-look results were reported in ATel \#3907 (Verrecchia et al. 2012). Performing a refined ML analysis by optimizing the background estimates on the AGILE-GRID data covering the seven day period from January 26 to February 2 (MJD 55952.5 to 55959.5 ), yields in a detection at a significance level of about $7 \sigma$. The flare-I spectral analysis gives a photon index $\Gamma=2.17 \pm 0.24$ and a flux $F(E>100 \mathrm{MeV})=(2.0 \pm 0.5) \times 10^{-6} \mathrm{ph} \mathrm{cm}^{-2} \mathrm{~s}^{-1}$.

The second flare (flare-II), with higher $\gamma$-ray flux, was announced with ATel \#3934 (Lucarelli et al. 2012). The source maintained its high state above $4.0 \times 10^{-6} \mathrm{ph} \mathrm{cm}^{-2} \mathrm{~s}^{-1}$ for almost two weeks. We performed the AGILE ML analysis on this two-week period (from 2012 February 20 to 2012 March 05, MJD 55977.5 to 55991.5 ) obtaining a detection at a $\sim 16 \sigma$ significance level. The corresponding spectral analysis provides a photon index $\Gamma=2.21 \pm 0.11$, consistent with that of Flare-I, but a higher flux $F(E>100 \mathrm{MeV})=(4.4 \pm 0.5) \times 10^{-6} \mathrm{ph} \mathrm{cm}^{-2} \mathrm{~s}^{-1}$.

After 2012 March 9 (MJD 55995) the source went back to a low-flux state, with the source sky position approaching the border of field of view of AGILE, and after 2012 March 14 (MJD 56000) the AGILE daily effective exposure gradually decreased. The ML analysis over the 14 day period starting on 2012 March 12 (MJD 55998.5) gives the source at a significance level of around $6 \sigma$, with a photon index $\Gamma=2.4 \pm$ 0.4 and an average flux $F(E>100 \mathrm{MeV})=(1.8 \pm 0.5) \times$ $10^{-6} \mathrm{ph} \mathrm{cm}^{-2} \mathrm{~s}^{-1}$.

\footnotetext{
ADC pointing (sw=5_19_18_17) and spinning (sw=5_21_18_19) archives, from http://agile.asdc.asi.it

2 AGILE_SW_5.0_SourceCode from ADC website, with I0023 calibrations.
} 
Table 1. Integral photon fluxes $>100 \mathrm{MeV}$ detected by AGILE-GRID.

\begin{tabular}{lcccc}
\hline \hline \multicolumn{1}{c}{ Epoch } & $\begin{array}{c}\text { Integration period } \\
{[\mathrm{MJD}]}\end{array}$ & $\begin{array}{c}\text { Energy bin } \\
{[\mathrm{MeV}]}\end{array}$ & $\begin{array}{c}\text { Flux } \\
{\left[\mathrm{ph} \mathrm{cm}^{-2} \mathrm{~s}^{-1}\right]}\end{array}$ & $\Gamma$ \\
\hline Flare-I (7 days) & $55952.5-55959.5$ & $>100$ & $(2.0 \pm 0.5) \times 10^{-6}$ & $2.17 \pm 0.24$ \\
Flare-II (14 days) & $55977.5-55991.5$ & $>100$ & $(4.4 \pm 0.5) \times 10^{-6}$ & $2.21 \pm 0.11$ \\
Postflare (14 days) & $55998.5-56012.5$ & $>100$ & $(1.8 \pm 0.5) \times 10^{-6}$ & $2.39 \pm 0.36$ \\
Low/intermediate state & $55746.5-55803.5$ & $>100$ & $(9.1 \pm 1.5) \times 10^{-7}$ & $2.44 \pm 0.17$ \\
\hline
\end{tabular}

Table 2. Differential flux values $(v \mathrm{~F}(v))$ detected by AGILE-GRID in 2012.

\begin{tabular}{ccccc}
\hline \hline Epoch & $\begin{array}{c}\text { Integration period } \\
{[\mathrm{MJD}]}\end{array}$ & $\begin{array}{c}\text { Energy bin } \\
{[\mathrm{MeV}]}\end{array}$ & $\begin{array}{c}v \\
{[\mathrm{~Hz}]}\end{array}$ & $\begin{array}{c}v F(v) \\
{\left[\mathrm{erg} \mathrm{cm}^{-2} \mathrm{~s}^{-1}\right]}\end{array}$ \\
\hline Flare-II (14 days) & $55977.5-55991.5$ & $100-200$ & $3.42 \times 10^{22}$ & $(7.0 \pm 1.1) \times 10^{-10}$ \\
& & $200-400$ & $6.85 \times 10^{22}$ & $(7.2 \pm 1.3) \times 10^{-10}$ \\
& & $400-10000$ & $4.84 \times 10^{23}$ & $(5.2 \pm 1.1) \times 10^{-10}$ \\
\hline Low/intermediate state & $55746.5-55803.5$ & $100-200$ & $3.42 \times 10^{22}$ & $(1.7 \pm 0.4) \times 10^{-10}$ \\
& & $200-400$ & $6.85 \times 10^{22}$ & $(1.7 \pm 0.4) \times 10^{-10}$ \\
& & $400-10000$ & $4.84 \times 10^{23}$ & $(6.4 \pm 0.3) \times 10^{-11}$ \\
\hline
\end{tabular}

For comparison, we have identified one of the typical low/intermediate states of the source with $\gamma$-ray flux below $10^{-6} \mathrm{ph} \mathrm{cm}-2 \mathrm{~s}^{-1}$, from 2011 July 4 to 2011 August 30 (MJD 55746.5 to 55803.5 ), and performed the AGILE ML analysis getting a photon index $\Gamma=2.44 \pm 0.17$ and a flux $F(E>100 \mathrm{MeV})=(0.91 \pm 0.15) \times 10^{-6} \mathrm{ph} \mathrm{cm}^{-2} \mathrm{~s}^{-1}$. AGILE results during the MAGIC observation period in 2012 and this low intermediate state are summarised in Tables 1 and 2.

\subsection{Fermi-LAT}

Fermi-Large Area Telescope (Fermi-LAT) is a pair conversion telescope designed to cover the energy band from $20 \mathrm{MeV}$ to greater than $300 \mathrm{GeV}$ (Atwood et al. 2009). In its primary observation strategy, survey mode, the LAT scans the entire sky every three hours and therefore can provide observations of PKS 1510-089 simultaneous to MAGIC.

PKS 1510-089 has been continuously monitored by Fermi and the data used for this analysis were collected from 2012 January 1 to April 7 (MJD 55927-56024). They were analysed with the standard analysis tool gtlike, part of the Fermi ScienceTools software package (version 09-27-01). Only good quality events within $10^{\circ}$ of PKS 1510-089 were selected for analysis. Moreover, to reduce the contamination from the Earthlimb $\gamma$ rays produced by cosmic ray interactions with the upper atmosphere, data were restricted to a maximal zenith angle of $100^{\circ}$ and time periods when the spacecraft rocking angle exceeded $52^{\circ}$ were excluded.

To extract the spectral information we used the standard background models provided by the publicly available files gal_2yearp7v6_v0_trim.fits and iso_p7v6source.txt ${ }^{3}$. The background templates, whose normalizations were left free during the fitting process, take into account the diffuse $\gamma$-ray emission from our Galaxy and an isotropic diffuse component. During the spectral fitting of the point source the normalizations of the components comprising the entire background model were allowed to vary freely. To derive the source spectral information an unbinned maximum likelihood technique was applied to events in the energy range from $100 \mathrm{MeV}$ to $300 \mathrm{GeV}$ (Mattox et al. 1996) in combination with the post-launch instrument response

\footnotetext{
3 http://fermi.gsfc.nasa.gov/ssc/data/access/lat/ BackgroundModels.html
}

functions P7SOURCE_V6. Sources from the 2FGL catalogue (Nolan et al. 2012) located within $15^{\circ}$ of PKS 1510-089 were incorporated in the model of the region by setting the spectral models and the initial parameters for the modelling to those reported in the 2FGL catalogue. In particular, the source of interest was modelled with a Log parabola spectrum ${ }^{4}$ :

$\frac{\mathrm{d} N}{\mathrm{~d} E}=N_{0}\left(\frac{E}{E_{\mathrm{b}}}\right)^{-\left(\alpha+\beta \log \left(\frac{E}{E_{\mathrm{b}}}\right)\right)}$

where $N_{0}$ is the normalization, $E_{\mathrm{b}}$ the break energy and $\alpha$ and $\beta$ parameters for the log parabola fit.

In the fitting procedure the parameters of sources located within a $10^{\circ}$ radius centred on the source of interest were left free to vary while parameters of sources located within a $10^{\circ}-15^{\circ}$ annulus were fixed. When performing the fit for the light curve and SED bins, the photon indices of the sources were frozen to the best-fit values obtained from a long-term analysis. Systematic uncertainties in LAT results due to uncertainties in the effective area are discussed in Ackermann et al. (2012); they are smaller than the statistical uncertainties of the points in the light curves and have been neglected.

The Fermi-LAT one day bin light curve is shown together with the multi-frequency light curves in Sect. 7. Since the source is not always significantly resolved, flux upper limits at $95 \%$ confidence level were calculated for each time bin where the test statistic $^{5}$ (TS) value for the source was TS $<25$. The light curve shows that the flaring activity had a duration of about 55 days in $\gamma$ rays and consisted of several distinct flares.

As PKS 1510-089 is known to show variability on time scale less than a day (Saito et al. 2013; Brown et al. 2013) we also searched for shorter time scale of variability within the brightest flaring epoch 2012 February 17 to March 8 (MJD 55 97455994 ) and produced light curves in bins of $1.5 \mathrm{~h}$ and $3 \mathrm{~h}$ (the latter is shown in Fig. 3). We systematically looked at the light curves and calculated the doubling times $\left(t_{\mathrm{d}}\right)$ between significant $(\mathrm{TS}>25)$ adjacent bins following $t_{\mathrm{d}}=\Delta t \ln 2 / \ln \left(F_{\max } / F_{\min }\right)$.

\footnotetext{
4 http://fermi.gsfc.nasa.gov/ssc/data/analysis/
} scitools/source_models.html

5 A maximum likelihood test statistic TS $=2 \Delta \log ($ likelihood) between models with and without a point source at the position of PKS 1510089 (Mattox et al. 1996). 
Table 3. Comparison of the different spectral models for the Fermi-LAT data for PKS 1510-089.

\begin{tabular}{|c|c|c|c|c|c|c|c|c|c|c|}
\hline \multirow[t]{2}{*}{ Epoch } & \multicolumn{4}{|c|}{ Power-law } & \multicolumn{5}{|c|}{ Log parabola } & \multirow[t]{2}{*}{$\sigma^{b}$} \\
\hline & Flux $^{a}$ & Index & TS & Loglike & Flux $^{a}$ & Alpha & Beta & TS & Loglike & \\
\hline $\begin{array}{l}\text { MAGIC } \\
\text { observation }\end{array}$ & $3.97 \pm 0.08$ & $2.39 \pm 0.02$ & 12241 & 107077 & $3.82 \pm 0.08$ & $2.24 \pm 0.03$ & $0.09 \pm 0.02$ & 12243 & 107056 & 6 \\
\hline Mean state & $2.67 \pm 0.04$ & $2.40 \pm 0.01$ & 19943 & 269334 & $2.56 \pm 0.04$ & $2.26 \pm 0.02$ & $0.09 \pm 0.01$ & 19942 & 269299 & 8 \\
\hline Low state & $0.79 \pm 0.04$ & $2.52 \pm 0.04$ & 1417 & 99964 & $0.75 \pm 0.04$ & $2.35 \pm 0.07$ & $0.12 \pm 0.04$ & 1422 & 99959 & 3 \\
\hline High state & $6.50 \pm 0.14$ & $2.29 \pm 0.02$ & 7389 & 41207 & $6.24 \pm 0.17$ & $2.12 \pm 0.04$ & $0.10 \pm 0.02$ & 7421 & 41191 & 4.5 \\
\hline
\end{tabular}

Notes. ${ }^{(a)}$ Flux $(100 \mathrm{MeV}-300 \mathrm{GeV})$ is in units of $\left[10^{-6} \mathrm{ph} \mathrm{cm}^{-2} \mathrm{~s}^{-1}\right]^{(b)}$ Significance by which the Log parabola model has to be preferred w.r.t. the simple power-law model $(\sigma)$ calculated as $\left[2\left(\log l i k e_{P w l}-\log l i k e_{\log P}\right)\right]^{1 / 2}$.

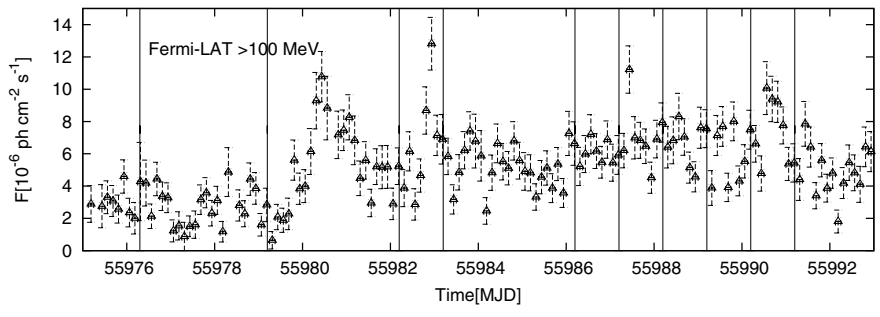

Fig. 3. Fermi-LAT $>100 \mathrm{MeV}$ light curve in the three hour bins for the first MAGIC observing period. The vertical lines represent the MAGIC observing times (all shorter than three hours in duration) showing that the MAGIC observation windows missed the times of the fastest HE $\gamma$-ray variability.

Excluding flux variations that were within $1 \sigma$ and doubling times with errors larger than $50 \%$, the shortest value that we derive for this period is $t_{\mathrm{d}}=1.5 \pm 0.6$ hour.

We considered the Fermi-LAT data of individual light-curve bins, fitting them with a power-law model in order to investigate spectral evolution in the HE range. In this analysis we do not find evidence for this behaviour, although we note that the source spectrum is better represented by the log parabola shape in several time intervals, thus the power-law fit may not adequately reproduce the source spectral shape. Additionally it is apparent that during the high state, the spectral index is significantly harder than for the low state or mean state (see below).

The SED was obtained combining all events of time intervals coincident with the last two VHE detections, i.e. from February 19 to March 5 (MJD 55976-55991) and from March 15 to April 3 (MJD 56001-56020). For comparison we analysed the mean state in 2012 January-April (MJD 55 927-56025), a low state SED which consists of the data taken in 2012 January and April (MJD 55927-55954 and 56007-56025) and a high state which consists of all time periods when the Fermi flux was $>6 \times 10^{-6} \mathrm{ph} \mathrm{cm}^{-2} \mathrm{~s}^{-1}$. The log parabola model is significantly preferred (in the MAGIC observing epoch with $6 \sigma$ significance and in the low state with $3 \sigma$ ) with respect to the powerlaw in all the time intervals considered for this SED analysis. The detailed results are shown in Table 3.

\subsection{Gamma-ray results}

We compared the results of the observations in HE and VHE $\gamma$ rays. As discussed in previous sections, the HE $\gamma$-ray flux is variable on time scales shorter than day. Therefore it appears that fast variability can explain the small mismatches between daily fluxes of Fermi-LAT and two-day fluxes by AGILE-GRID. These light curves are shown together with multi-frequency light curves in Sect. 7. The variability amplitude of the HE $\gamma$-ray flux is rather large (more than one order of magnitude in flux) in the first MAGIC observing period (MJD 55976 to 55991).
Still, within this period, MAGIC observed no statistically significant variability from the source. In Fig. 3 the Fermi-LAT light curve in three hour bins is shown. The vertical lines show the MAGIC observation times, revealing that the MAGIC observations missed all the periods of fast HE $\gamma$-ray variability and therefore it was to be expected that no fast variability would be detected in the MAGIC observations. Apparently the MAGIC observations also missed the highest peaks of the HE $\gamma$-ray light curve. The maximum flux measured simultaneous to the MAGIC observations is $F(>100 \mathrm{MeV}) \sim 8 \times 10^{-6} \mathrm{ph} \mathrm{cm}^{-2} \mathrm{~s}^{-1}$ and the average of the strictly simultaneous bins is $F(>100 \mathrm{MeV}) \sim$ $4.4 \times 10^{-6} \mathrm{ph} \mathrm{cm}^{-2} \mathrm{~s}^{-1}$.

For the second MAGIC observation window in March-April (from 56001 to 56020), fast variability could not be investigated because of the lower HE $\gamma$-ray state of the source. After March 23 (MJD 56009), the source was no longer detected on daily scales in HE $\gamma$ rays, the daily upper limits being below $1.0 \times 10^{-6} \mathrm{ph} \mathrm{cm}^{-2} \mathrm{~s}^{-1}$. Therefore, in total, the HE $\gamma$-ray flux variability amplitude, within the windows strictly simultaneous to the MAGIC observing windows, was eight on nightly scales, which could go undetected in the MAGIC light curve given the overall low flux as discussed in Sect. 2.2. It is therefore not possible to conclude if the lack of significant variability in the VHE $\gamma$-ray band has a real physical origin or if it is simply an observational bias (either due to unfortunate sampling or due to low photon statistics).

The SED of PKS $1510-089$ from $\sim 100 \mathrm{MeV}$ to $\sim 400 \mathrm{GeV}$ is presented in Fig. 4. The HE $\gamma$-ray data from AGILE-GRID and Fermi-LAT cover slightly different periods (AGILE from MJD 55977.5 to 55991.5 and Fermi-LAT from MJD 55976 to 55991 and from 56001 to 56020 ). The AGILE-GRID data consist of flaring state data only while the Fermi-LAT spectrum summarises all events of the time intervals coincident with the MAGIC observation window. As suggested by AGILE and confirmed by Fermi-LAT, the brighter states are characterised by a hardening of the HE spectrum, and therefore the higher flux observed by AGILE at $2 \mathrm{GeV}$ is expected. The peak of the SED is at $\sim 100 \mathrm{MeV}$. The log parabola fit and the errors of the FermiLAT spectra have been extrapolated to the MAGIC energy range. We also show the extrapolation taking into account the EBL absorption using the model of Dominguez et al. (2011). The VHE $\gamma$-ray spectrum observed by MAGIC connects smoothly with this extrapolation suggesting that the emission originates in the same region.

\section{Swift X-ray observations, data analysis and results}

The Swift satellite (Gehrels et al. 2004) performed 23 ToO observations on PKS 1510-089 between 2012 February 2 and April 5 (MJD 55959-56022), triggered by the strong activity of the 
Table 4. Log and fitting results of Swift-XRT observations.

\begin{tabular}{ccccc}
\hline \hline Date & $\begin{array}{c}\text { Net Exp. time } \\
(\mathrm{s})\end{array}$ & $\begin{array}{c}\text { Photon index } \\
\Gamma\end{array}$ & $\begin{array}{c}\text { Flux 0.3-10.0 keV } \\
\left(\times 10^{-12} \mathrm{erg} \mathrm{cm}^{-2} \mathrm{~s}^{-1}\right)\end{array}$ & $\chi_{\text {red }}^{2}$ (d.o.f.) / Cash \\
\hline $2012-02-02$ & 2470 & $1.35 \pm 0.17$ & $7.8 \pm 0.7$ & Cash \\
$2012-02-04$ & 2450 & $1.42 \pm 0.16$ & $10.7 \pm 1.2$ & $0.85(19)$ \\
$2012-02-05$ & 2655 & $1.27 \pm 0.16$ & $10.1 \pm 1.1$ & $1.00(18)$ \\
$2012-02-07$ & 2140 & $1.56 \pm 0.16$ & $8.0 \pm 1.2$ & $1.00(14)$ \\
$2012-02-17$ & 789 & $1.65 \pm 0.21$ & $8.7 \pm 1.7$ & Cash \\
$2012-02-19$ & 5781 & $1.63 \pm 0.09$ & $7.9 \pm 0.6$ & $0.95(39)$ \\
$2012-02-21$ & 1286 & $1.60 \pm 0.23$ & $8.6 \pm 1.5$ & $0.76(8)$ \\
$2012-02-22$ & 2700 & $1.51 \pm 0.14$ & $9.0 \pm 1.0$ & $1.05(19)$ \\
$2012-02-23$ & 2989 & $1.43 \pm 0.13$ & $9.6 \pm 1.1$ & $0.85(22)$ \\
$2012-03-01$ & 1024 & $1.37 \pm 0.18$ & $10.8 \pm 1.7$ & Cash \\
$2012-03-18$ & 3224 & $1.36 \pm 0.13$ & $11.6 \pm 1.3$ & $0.77(20)$ \\
$2012-03-20$ & 1351 & $1.45 \pm 0.17$ & $8.9 \pm 1.5$ & Cash \\
$2012-03-22$ & 2477 & $1.28 \pm 0.21$ & $8.6 \pm 1.6$ & $1.06(9)$ \\
$2012-03-24$ & 1219 & $1.31 \pm 0.17$ & $12.5 \pm 1.9$ & Cash \\
$2012-03-30$ & 2695 & $1.58 \pm 0.13$ & $7.9 \pm 0.9$ & $1.01(17)$ \\
$2012-04-01$ & 2620 & $1.59 \pm 0.14$ & $8.6 \pm 0.9$ & $0.71(17)$ \\
$2012-04-03$ & 1596 & $1.40 \pm 0.15$ & $9.3 \pm 1.2$ & Cash \\
$2012-04-05$ & 1196 & $1.61 \pm 0.20$ & $7.1 \pm 1.2$ & Cash \\
\hline
\end{tabular}

Notes. ${ }^{(a)}$ Observed flux.

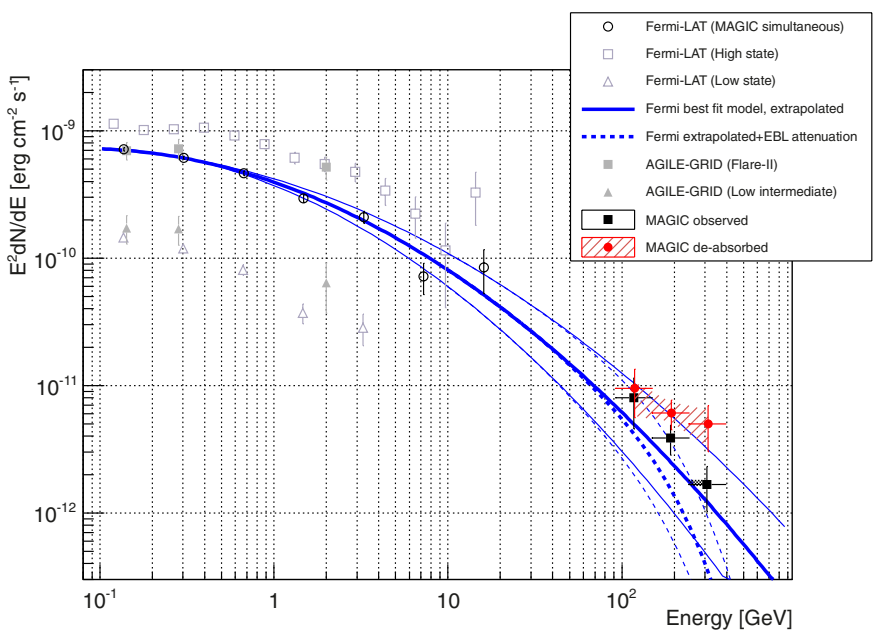

Fig. 4. $\gamma$-ray SED constructed from AGILE, Fermi-LAT and MAGIC data. The AGILE-GRID data (grey filled squares) correspond to the data of Flare-II (from MJD 55 977.5 to 55 991.5). The Fermi-LAT spectrum (black open circles) combines all events of time intervals coincident with the MAGIC observation window (MJD 55976 to 55991 and from 56001 to 56020) with the blue lines showing the log parabola fit to the data and its statistical uncertainty (the thinner lines). The fit and the errors of the Fermi-LAT spectra have been extrapolated to MAGIC energy range. The dashed blue lines show the extrapolation with the EBL absorption effects included. The MAGIC data points are shown with black filled squares (observed) and red filled circles (de-absorbed). The corresponding shaded region indicates the statistical uncertainty of the spectral fitting (same as in the Fig. 2). The grey data shows, for the comparison, the low-intermediate state spectrum of the source as measured by AGILE-GRID (triangles) and Fermi-LAT (open triangles) and high-state SED as measured by Fermi-LAT (open squares).

source detected first by AGILE (Lucarelli et al. 2012) and FermiLAT at HE $\gamma$-ray energies, and then by MAGIC at TeV energies (Cortina 2012). The observations were performed with all three onboard instruments: the X-ray Telescope (XRT; Burrows et al. 2005, 0.2-10.0 keV), the Ultraviolet Optical Telescope (UVOT; Roming et al. 2005, 170-600 nm), and the Burst Alert Telescope (BAT; Barthelmy et al. 2005, 15-150 keV).
For the Swift-XRT data analysis, we considered observations with exposure times longer than 500 seconds, including $20 \mathrm{ob}-$ servations. In addition we summed the data of the three observations performed on February 19 in order to have higher statistics. The XRT data were processed with standard procedures (xrtpipeline vQ.12.6), filtering, and screening criteria by using the Heasoft package (v6.11). The source count rate was low during the entire campaign $\left(<0.5\right.$ counts $\left.\mathrm{s}^{-1}\right)$, so we only considered photon counting data and further selected XRT event grades 0-12. Pile-up correction was not required. Source events were extracted from a circular region with a radius of 20 pixels (one pixel $\sim 2.36^{\prime \prime}$ ), while background events were extracted from a 50 pixel radius circular region not containing any contaminating sources and lying away from the source region. The spectral redistribution matrices v013 in the Calibration database maintained by HEASARC were used.

The adopted energy range for spectral fitting is $0.3-10 \mathrm{keV}$. When the number of counts was less than 200 the Cash statistic (Cash 1979) on ungrouped data was used. All the other spectra were rebinned with a minimum of 20 counts per energy bin to allow $\chi^{2}$ fitting within XSPEC (v12.6.0; Arnaud 1996). We fitted the individual spectra with a simple absorbed power law, with a neutral hydrogen column density fixed to its Galactic value $\left(6.89 \times 10^{20} \mathrm{~cm}^{-2}\right.$; Kalberla et al. 2005). The fit results are reported in Table 4.

During the observations Swift-XRT detected the source with a flux, $F(0.3-10 \mathrm{keV})$, in the range $(0.7-1.2) \times$

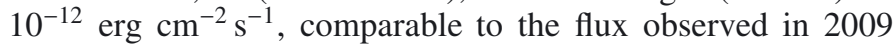
March, during a period of high HE $\gamma$-ray activity (D'Ammando et al. 2011; Abdo et al. 2010a), but lower with respect to the high flux level observed in 2006 August (Kataoka et al. 2008). The light curve is shown in Sect. 7, together with the multi-frequency data.

The flux versus photon index plot is shown in Fig. 5. At higher flux the photon index seems to become harder. This behaviour is consistent with the harder when brighter trend reported in Kataoka et al. (2008) and D'Ammando et al. (2011). As discussed in these papers, such a trend indicates that in bright states the X-ray emission is completely dominated by external 


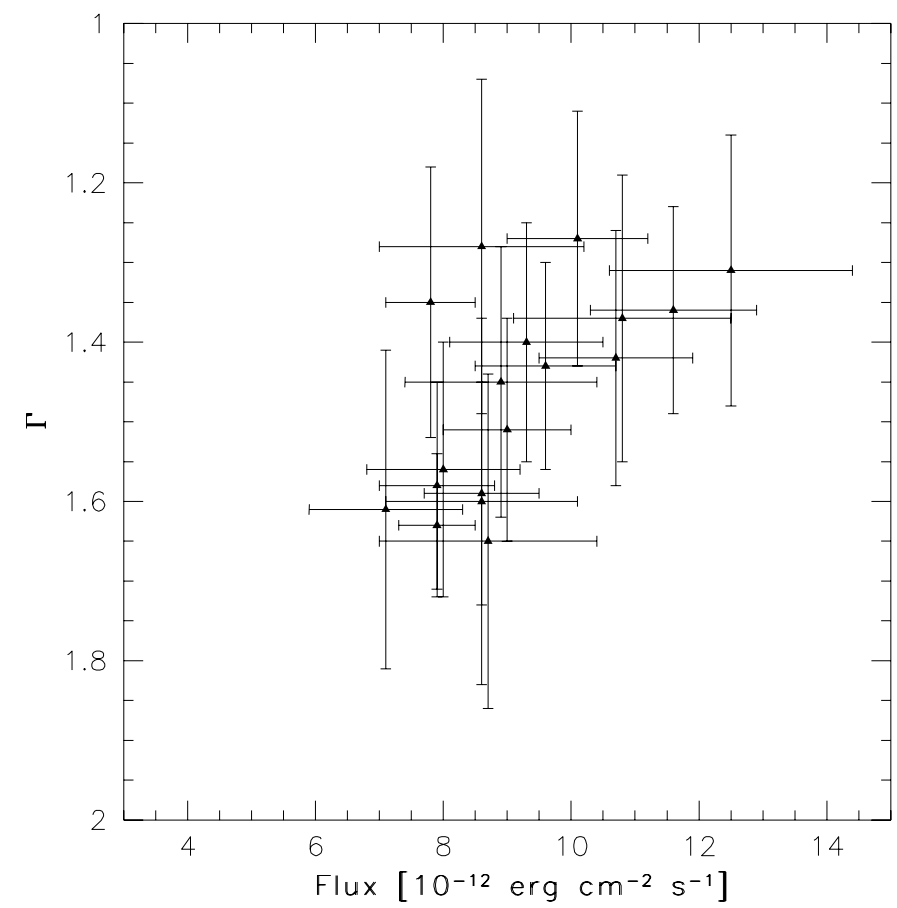

Fig. 5. Flux $(0.3-10 \mathrm{keV})$ versus photon index for Swift-XRT. Although there was only marginal X-ray variability during the observations, the plot shows a hint of harder when brighter trend.

Compton emission, while in lower state there is also contribution from a soft excess component, which could be e.g. a blurred reflection, Comptonization of the thermal disc emission or a mixture of synchrotron, external Compton and SSC emission.

We also investigated the Swift-BAT data using the SwiftBAT Hard X-ray Transient Monitor (Krimm et al. 2013). In the BAT data for 2012 January-April there is only a hint of signal $(2.5 \sigma)$ on 2012 February 9 (MJD 55966), with a rate of $(0.0033 \pm 0.0013)$ counts s${ }^{-1} \mathrm{~cm}^{-2}$, corresponding to $15 \mathrm{mCrab}$ in the $15-50 \mathrm{keV}$ energy band. As a comparison, in 2009 March the high flux observed by BAT in hard X-ray was $40 \mathrm{mCrab}$ (D’Ammando et al. 2011).

\section{Ultraviolet, optical, near infrared observations, data analysis, and results}

PKS 1510-089 is included in many ongoing optical blazar monitoring programmes which provide good coverage from ultraviolet (UV) to infrared (IR) bands (Fig. 6.). Polarimetric observations of the source were also performed. The participating observatories are described in Sects. 5.1-5.6 and the results of the optical observations are discussed in Sect. 5.7.

\subsection{Ultraviolet and optical photometry from UVOT}

The UVOT covers the $180-600 \mathrm{~nm}$ wavelength range using filters: $U V W 2, U V M 2, U V W 1, U, B$ and $V$ (Poole et al. 2008). We reduced the Swift-UVOT data with the Heasoft package version 6.12 and the 20111031 release of the Swift-UVOTA CALDB. Multiple exposures in the same filter at the same epoch were summed with uvotimsum, and aperture photometry was then performed with the task uvotsource. Source counts were extracted from a circular region with a 5 arcsec radius centred on the source. Background counts were estimated in a surrounding annulus with inner and outer radii of 15 and 25 arcsec, respectively. The background region was selected such that it does not contain any contaminating sources.

We also compiled SEDs for all 19 epochs for which observations in all the six UVOT filters were available. The $\lambda_{\text {eff }}$ and count-rate-to-flux conversion factors were derived by convolving the source spectrum with the effective areas of the UV filters. In the same way we calculated the Galactic extinction in the various bands, using the Cardelli et al. (1989) law and setting $R_{V}=3.1$ and $A_{B}=0.416$ after Schlegel et al. (1998). The results were used to obtain de-reddened flux densities. Four out of the 19 SEDs (for the sake of clarity) were combined with the optical and IR data and are shown in Fig.7. These epochs correspond to pre-outburst (2012 February 7, MJD 55 964), two local maxima (2012 February 24, MJD 55981 and 2012 March 1, MJD 55 987) and post-outburst (2012 March 26, MJD 56012) phases of the light curves.

\subsection{Optical $R$-band photometry from KVA}

PKS 1510-089 was observed as a part of the Tuorla blazar monitoring programme ${ }^{6}$, which provides optical support observations for the MAGIC telescopes and participates in the GASP-WEBT collaboration, with the KVA $35 \mathrm{~cm}$ telescope at Observatorio del Roque de los Muchachos, La Palma. The observations started on 2012 January 14 (MJD 55 940) and after 2012 February 2 (MJD 55959 ), the source was observed every night, weather and moon conditions allowing. The data were reduced using the standard data analysis pipeline (Nilsson et al., in prep.) and the fluxes were measured with differential photometry, using the comparison stars from Villata et al. (1997).

\subsection{Optical photometry and polarisation from Steward and Perkins Observatories}

Optical (4000-7550 $\AA$ ) spectropolarimetry and differential spectrophotometry were performed at the Steward Observatory $2.3 \mathrm{~m}$ Bok Telescope using the SPOL CCD Imaging/Spectropolarimeter. These observations were obtained as part of an ongoing monitoring programme of $\gamma$-ray bright blazars in support of the $\mathrm{Fermi}^{7}$.

The observations took place on 2012 January 22-29, 2012 February 13-21 and 2012 March 21-28 (MJD 55 948-55955, 55 970-55978, 56 007-56014). The data analysis pipeline is described in Smith et al. (2009).

Polarimetric and photometric $R$-band observations were also provided by the $1.8 \mathrm{~m}$ Perkins telescope of Lowell Observatory equipped with PRISM (Perkins Reimaging System) in 2012 March. The data analysis was done following the standard procedures as in Chatterjee et al. (2008).

Because the EVPA has a $\pm 180^{\circ} \times n($ where $n=1,2, \ldots)$ ambiguity, we selected the values such that the differences between any two points were minimised. There was one data point (Fig. 6) which differed by $\sim 90^{\circ}$ from the previous observation; we thus selected the EVPA for this point which does not cause a change in the direction of rotation between the two points.

\subsection{Optical and near infrared observations from GASP-WEBT}

Additional $R$-band monitoring data were collected by the GLAST-AGILE support programme (GASP) of the Whole

\footnotetext{
6 http://users.utu.fi/kani/1m

7 http://james.as.arizona.edu/ psmith/Fermi
} 


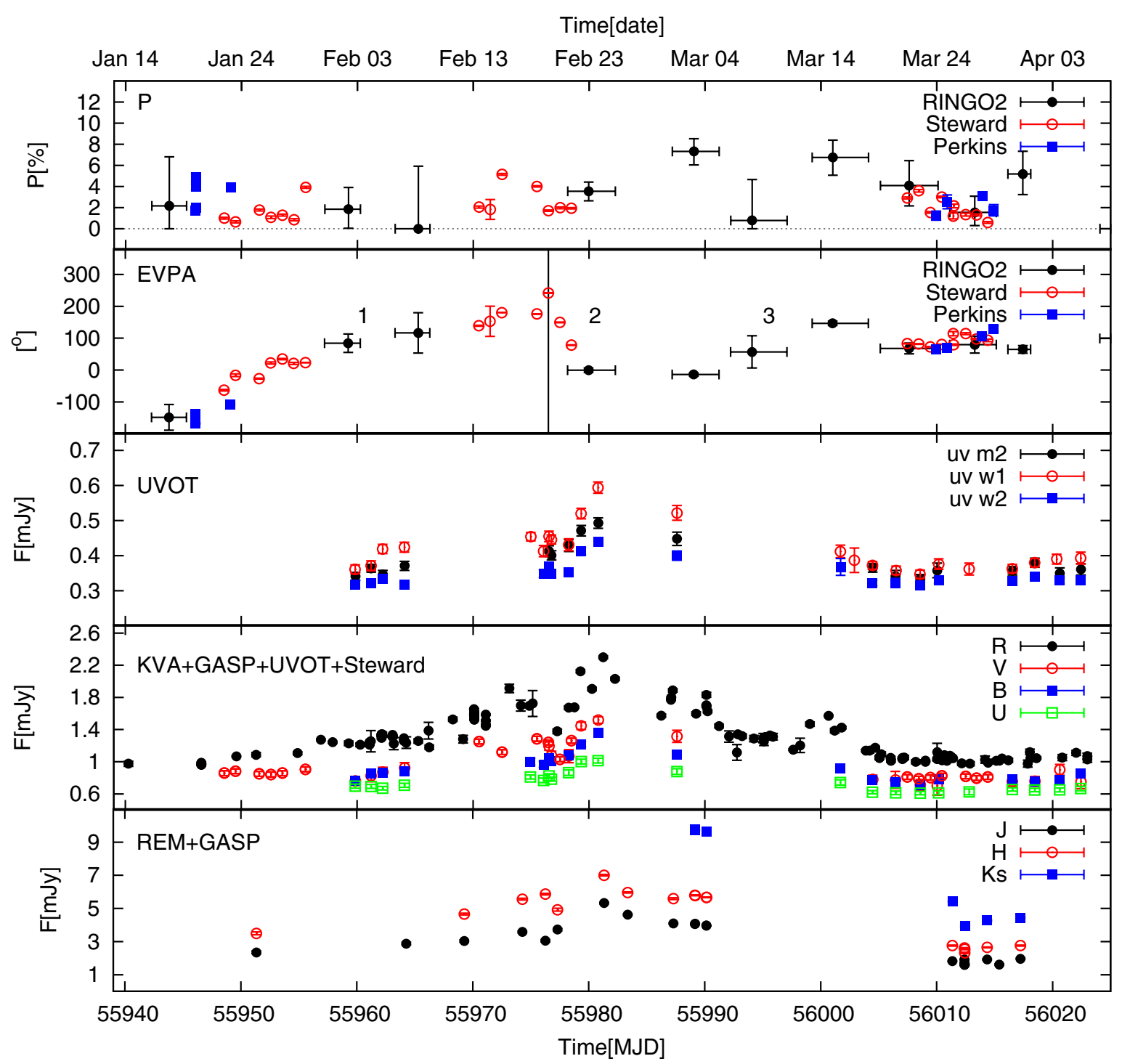

Fig. 6. Light curves of PKS 1510-089 in the UV, optical and near IR bands. The optical polarisation degree and angle are also shown in the two top panels. The next panels show UV (Swift-UVOT, middle), optical (KVA/GASP/UVOT, second from bottom) and near IR (REM and GASP, bottom) light curves of the source. The numbers in the second from the top panel refers to the rotations of the EVPA discussed in the text. Vertical line indicates the time when the PA changes by $\sim 90^{\circ}$ between the highlighted point and the previous point (see text). The fluxes are given in mJy and are not corrected for Galactic absorption.

Earth Blazar Telescope ${ }^{8}$ (WEBT). These GASP observations of PKS 1510-089 were performed by the following observatories: Abastumani, Calar Alto, Crimean, Lulin, Rozhen, St. Petersburg and Teide. The source magnitude is calculated with respect to the reference stars two to six calibrated by Raiteri et al. (1998). The GASP near IR data were acquired in the $J, H$, and $K$ s bands with the IAC80 and Carlos Sanchez telescopes at Teide Observatory. Their calibration was performed using field stars with the most reliable photometry (signal to noise ratio, $S / N \gtrsim 10$ and uncertainty $\sigma<0.11$ ) in the Two Micron All Sky Survey ${ }^{9}$ (2MASS) catalogue.

\subsection{Near infrared observations from REM}

REM (Rapid Eye Mount) is a $60 \mathrm{~cm}$ diameter fast reacting telescope located at La Silla, Chile. The telescope has two instruments: REMIR, an infrared imaging camera, and ROSS,

\footnotetext{
8 http://www.oato.inaf.it/blazars/webt

9 http://www.ipac.caltech.edu/2mass/
}

a visible imager and slitless spectrograph (Zerbi et al. 2001; Chincarini et al. 2003; Covino et al. 2004a,b). PKS 1510-089 was observed by REM starting on 2012 January 25 (MJD 55951) during 28 nights. Typical exposure durations were of $30 \mathrm{~s}$ in the $J, H$, and $K$ s filters. The data were analysed in a standard way using tools provided by the ESO-Eclipse package (Devillard 1997). Standard aperture photometry was derived and results calibrated by a suitable number of well-exposed 2MASS objects in the field ${ }^{9}$.

\subsection{Optical polarimetry observations from Liverpool Telescope}

RINGO-2 is a fast readout imaging polarimeter mounted in the fully robotic $2 \mathrm{~m}$ Liverpool Telescope at Observatorio del Roque de los Muchachos, La Palma. RINGO2 uses a hybrid $V+R$ filter, consisting of a $3 \mathrm{~mm}$ Schott GG475 filter cemented to a $2 \mathrm{~mm}$ $K G 3$ filter. PKS 1510-089 was observed as part of a monitoring programme and started on 2012 January 19 (MJD 55 945) with 


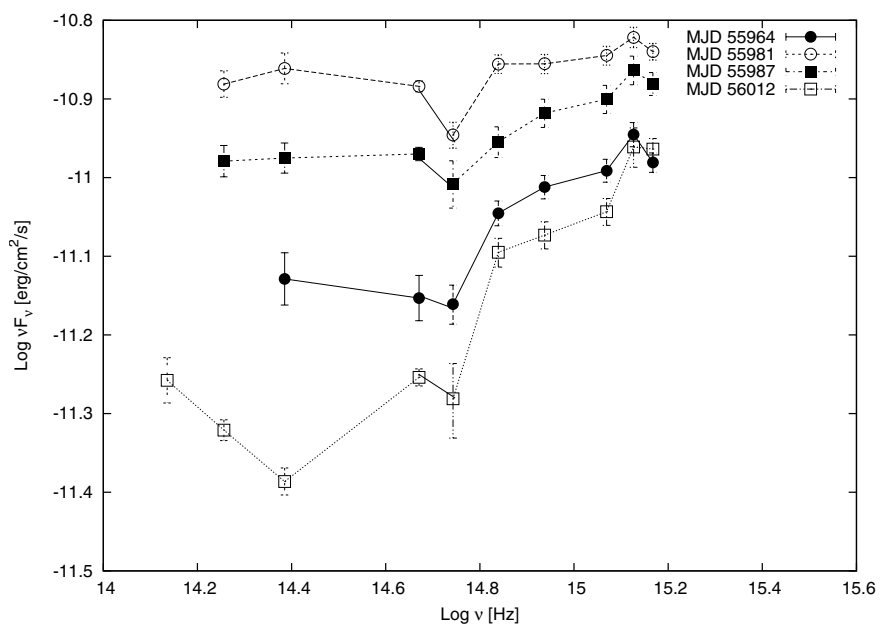

Fig. 7. Evolution of the infrared to ultraviolet SED from pre-outburst (MJD 55 964) to two local maxima (MJD 55981 and 55987) and to post-outburst (MJD 56 012) phase of the light curves. The data are corrected for Galactic absorption using Schlegel et al. (1998).

rather sparse sampling, but after 2012 February 21 (MJD 55 978) the source was observed every night, weather and moon conditions allowing. The data were reduced as described in Aleksić et al. (2014) using a data reduction pipeline written for the monitoring programme. Inspection of the data revealed that due to the combination of bright moon, partial cloud coverage and low average polarization of PKS $1510-089$, the $\mathrm{S} / \mathrm{N}$ was very low during many nights and no significant polarization was detected. In order to improve the $\mathrm{S} / \mathrm{N}$ we averaged observations over five day bins by first averaging $Q / I$ and $U / I$ and then computing the unbiased degree of polarization $p_{0}$ and its error as in Aleksić et al. (2014) with the difference that the error of EVPA was computed using the confidence intervals in Naghizadeh-Khouei \& Clarke (1993), which are better suited for low S/N data than the $\sigma(\mathrm{EVPA})=28.65 \times \sigma_{\mathrm{p}} / p$ formula used in Aleksić et al. (2014).

\subsection{Results}

The optical-UV and polarisation light curves from 2012 January to April (MJD 55 952-56025) are shown in Fig. 6. The light curves show an increasing flux peaking at near IR to UV wavelengths on 2012 February 25 (MJD 55 982), the optical flux more than doubles and reaches a maximum flux of $2.23 \pm 0.39 \mathrm{mJy}$ in the $R$-band. After that the general trend of the light curves is decreasing. On the top of this flare, the $R$-band light curve which is the best sampled light curve, shows several smaller amplitude $(<0.5 \mathrm{mJy})$ local minima and maxima. In particular there is a dip in the light curve on 2012 February 19 (MJD 55 976.5) and three local maxima after the major peak (2012 March 1, March 5 and March 13; MJD 55 987, 55990 and 55999). The flux densities varied by $5 \mathrm{mJy}(K \mathrm{~s}), 1.5 \mathrm{mJy}(R)$ and $0.2 \mathrm{mJy}(U V W 1)$. Hence, the source variability amplitude decreases as the frequency increases, as is usually found in FSRQs. This can be explained by the accretion disc emission diluting the UV emission from the jet (e.g. Raiteri et al. 2008, 2012) and the emission originating from the disc needs to be taken into account in the SED modelling (see Sect. 8).

The optical polarisation degree was generally low $\left(<10^{\circ}\right)$ during 2012 January-April compared to previous observations (e.g. Marscher et al. 2010). Therefore the error bars of the measurements are rather large. The EVPA showed three rotations of $>180^{\circ}$. The first one started in the beginning of the campaign and ended around 2012 February 20 (MJD 55 977, Fig. 6). The rotation was $\sim 380^{\circ}$, with a rotation rate of $\sim 10^{\circ} /$ day in counter-clockwise direction. The visual appearance of the rotation curve is rather smooth, but is rather poorly sampled between January 29 and February 13 (MJD 55955 and 55970). The second rotation started on February 20 (MJD 55 977) and ended on February 25 (MJD 55 982), lasting only five days. The rotation is $\sim 250^{\circ}$ and the direction is opposite to the first rotation (i.e. clockwise). After these two rotations the EVPA was stable at $\sim 0^{\circ}$ until March 7 (MJD 55993) when the third rotation started in a counter-clockwise direction and ended around March 14 (MJD 56 000) at $150^{\circ}$. On March 22 (MJD 56 008) it dropped to $\sim 80^{\circ}$ and remained stable until the end of the campaign.

The comparison of these rotations with the photometric light curve and polarisation degree behaviour shows that the first rotation takes place during an increase in the optical flux. The second rotation starts when there is a small dip in the optical $R$-band light curve and a local minimum in the polarisation degree. The rotation stops when the optical flare peaks. The third rotation starts with a small optical outburst and stops when the decay phase of the optical flare has reached a plateau.

We constructed SEDs from IR to UV for four distinct epochs: 2012 February 7 (MJD 55964, before the outburst), February 24 (MJD 55981, peak of the outburst), March 1 (MJD 55 987, second local maxima in the $R$-band light curve) and March 26 (MJD 56012, quiescent state after the outbursts), shown in Fig. 7. A softening of the SED from the pre-burst epoch to the epoch of outburst maxima is clearly visible. In the first and last SEDs, taken before and after the outburst, the thermal contributions from the accretion disc are again clearly visible as a strong increasing trend in the optical and UV bands. This behaviour was also seen for the 2009 outburst reported in D'Ammando et al. (2011).

\section{Radio observations, data analysis, and results}

PKS 1510-089 is part of the numerous blazar radio monitoring programmes extending from $2.6 \mathrm{GHz}$ to $230 \mathrm{GHz}$ by F-GAMMA, Medicina, UMRAO, OVRO, Metsähovi, VLBA and the Submillimeter Array. The observations collected for this paper are presented in Sects. 6.1-6.7 and the results discussed in Sect. 6.8.

\subsection{Submillimeter Array}

The $230 \mathrm{GHz}(1.3 \mathrm{~mm})$ light curve was obtained at the Submillimeter Array (SMA) on Mauna Kea (Hawaii). The SMA is an 8-element interferometer, consisting of $6 \mathrm{~m}$ dishes that may be arranged into configurations with baselines as long as $509 \mathrm{~m}$, producing a synthesised beam of sub-arcsecond width. PKS 1510-089 is included in an ongoing monitoring programme at the SMA to determine the fluxes of compact extragalactic radio sources that can be used as calibrators at $\mathrm{mm}$ wavelengths (Gurwell et al. 2007). Observations of available potential calibrators are usually observed for three to five minutes, and the measured source signal strength calibrated against known standards, typically solar system objects (Titan, Uranus, Neptune, or Callisto). Data from this programme are updated regularly and are available at the SMA website ${ }^{10}$.

${ }^{10}$ http://sma1.sma.hawaii.edu/callist/callist.html 


\subsection{Metsähovi radio telescope}

The $37 \mathrm{GHz}$ observations were made with the $13.7 \mathrm{~m}$ diameter Metsähovi radio telescope, which is a radome enclosed paraboloid antenna situated in Finland. The measurements were made with a $1 \mathrm{GHz}$-band dual beam receiver centred at $36.8 \mathrm{GHz}$. The beamwidth is $2.4 \mathrm{arcmin}$. The high electron mobility pseudomorphic transistor front end operates at room temperature. The observations were performed in an $\mathrm{ON}-\mathrm{ON}$ configuration alternating the source in each feed horn, with the second horn observing the sky. The flux density scale was set by observations of calibrator DR 21. The sources NGC 7027, 3C 274 and 3C 84 were used as secondary calibrators. A detailed description of the data reduction and analysis is given in Teräsranta et al. (1998). The error estimate in the flux density includes contributions from the measurement rms and the uncertainty of the absolute calibration. The PKS 1510-089 observations were done as part of the regular monitoring programme and the GASP-WEBT campaign.

\subsection{Owens Valley Radio Observatory}

Regular 15 GHz observations of PKS 1510-089 were carried out as part of a high-cadence $\gamma$-ray blazar monitoring programme using the Owens Valley Radio Observatory (OVRO) $40 \mathrm{~m}$ telescope in Owens Valley, California (Richards et al. 2011). This programme, which commenced in late 2007, now includes about 1800 sources, each observed with a nominal twice per week cadence.

The OVRO $40 \mathrm{~m}$ uses off-axis dual-beam optics and a cryogenic high electron mobility transistor low-noise amplifier with a $15.0 \mathrm{GHz}$ centre frequency and $3 \mathrm{GHz}$ bandwidth. The telescope and receiver combination produces a pair of approximately Gaussian beams (157 arcsec full width half maximum; FWHM), separated in azimuth by 12.95 arcmins. The total system noise temperature is about $52 \mathrm{~K}$, including receiver, atmosphere, ground, and CMB contributions. The two sky beams were Dicke switched using the OFF-source beam as a reference, and the source is alternated between the two beams in an ON-ON fashion to remove atmospheric and ground contamination. A noise level of approximately 3-4 mJy in quadrature with about $2 \%$ additional uncertainty, mostly due to pointing errors, is achieved in a $70 \mathrm{~s}$ integration period. Calibrations were performed using a temperature-stable diode noise source to remove receiver gain drifts and the flux density scale was derived from observations of 3C 286 assuming the Baars et al. (1977) value of $3.44 \mathrm{Jy}$ at $15.0 \mathrm{GHz}$. The systematic uncertainty of about $5 \%$ in the flux density scale is not included in the error bars. Complete details of the reduction and calibration procedure are found in Richards et al. (2011).

\subsection{F-GAMMA programme}

The $\mathrm{cm} / \mathrm{mm}$ radio light curves of PKS 1510-089 have been obtained within the framework of a Fermi-GST related monitoring programme of $\gamma$-ray blazars (F-GAMMA programme, Fuhrmann et al. 2007; Angelakis et al. 2008). The overall frequency range spans from $2.64 \mathrm{GHz}$ to $142 \mathrm{GHz}$ using the $100 \mathrm{~m}$ radio telescope located in Effelsberg, Germany and IRAM $30 \mathrm{~m}$ located on Pico Veleta in the Spanish Sierra Nevada.

The Effelsberg measurements were conducted with the secondary focus heterodyne receivers at $2.64,4.85,8.35,10.45$, $14.60,23.05,32.00$ and $43.00 \mathrm{GHz}$. The observations were performed quasi-simultaneously with cross-scans, slewing in azimuth and elevation across the source position with an adaptive number of sub-scans until the desired sensitivity is reached (for details, see Fuhrmann et al. 2008; Angelakis et al. 2008). Consequently, pointing off-set correction, gain correction, atmospheric opacity correction and sensitivity correction have been applied to the data.

The IRAM $30 \mathrm{~m}$ observations were carried out with calibrated cross-scans using the new EMIR horizontal and vertical polarisation receivers operating at 86.2 and $142.3 \mathrm{GHz}$. The opacity corrected intensities were converted into the standard temperature scale and finally corrected for small remaining pointing offsets and systematic gain-elevation effects. The conversion to the standard flux density scale was done using the instantaneous conversion factors derived from frequently observed primary (Mars, Uranus) and secondary $(\mathrm{W} 3(\mathrm{OH})$, K3-50A, NGC 7027) calibrators.

\subsection{UMRAO}

Centimetre band total flux density observations were obtained with the University of Michigan Radio Observatory (UMRAO) $26 \mathrm{~m}$ paraboloid located in Dexter, Michigan, USA. The instrument is equipped with transistor-based radiometres operating at frequencies centred at 4.8, 8.0, and $14.5 \mathrm{GHz}$ with bandwidths of $0.68,0.79$, and $1.68 \mathrm{GHz}$, respectively. Dual horn feed systems are used at 8 and $14.5 \mathrm{GHz}$, while at $4.8 \mathrm{GHz}$ a single-horn, mode-switching receiver is employed. Each observation consisted of a series of 8 to 16 individual measurements over approximately a 25 to 45 minutes time period, utilizing an ON-OFF observing technique at $4.8 \mathrm{GHz}$, and an ON-ON technique (switching the target source between the two feed horns which are closely spaced on the sky) at 8.0 and $14.5 \mathrm{GHz}$. As part of the observing procedure, drift scans were made across strong sources to verify the telescope pointing correction curves, and observations of nearby calibrators were obtained every one to two hours to correct for temporal changes in the antenna aperture efficiency. The PKS 1510-089 observations were done as part of the regular monitoring programme and the GASP-WEBT campaign.

\subsection{Medicina}

The Medicina telescope is a $32 \mathrm{~m}$ parabolic antenna located $30 \mathrm{~km}$ from Bologna, Italy, performing observations at both 5 and $8.4 \mathrm{GHz}^{11}$. FWHM beamwidth is $38.7 \mathrm{arcmin} /$ frequency $(\mathrm{GHz})$. We used the new enhanced single-dish control system acquisition system, which provides enhanced sensitivity and supports observations with the cross scan technique. All observations were performed at both 5 and $8.4 \mathrm{GHz}$; the typical onsource time is $1.5 \mathrm{~min}$ and the flux density was calibrated with respect to 3C 286. PKS 1510-089 was observed during 2012 January-April as part of the regular monitoring programme and the GASP-WEBT campaign.

\subsection{Very Long Baseline Array}

VLBA is a system of ten radio-telescope antennas, each with a dish $25 \mathrm{~m}$ in diameter located from Mauna Kea in Hawaii to St. Croix in the US Virgin Islands. VLBA observations

${ }^{11}$ http://www.med.ira.inaf.it/parabola_page_EN.htm 


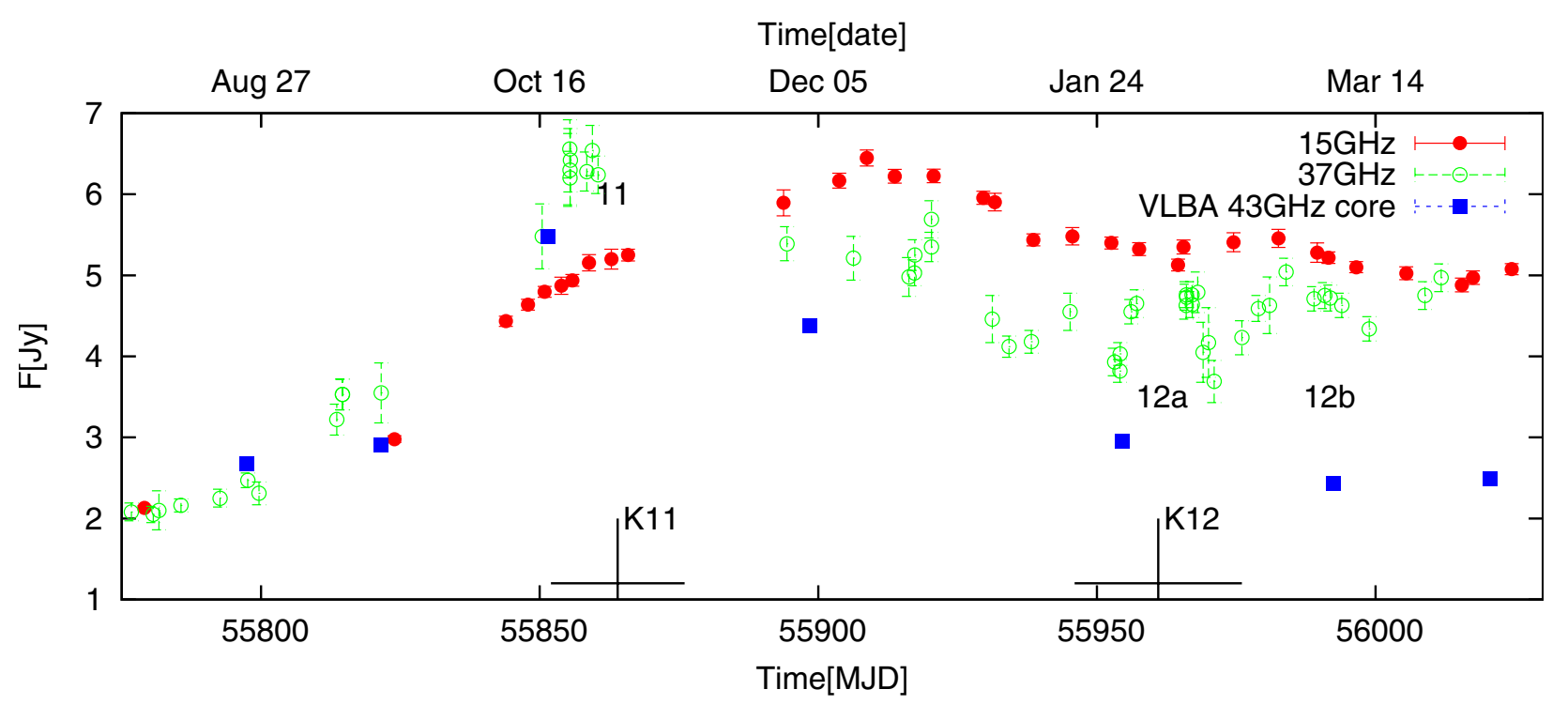

Fig. 8. $15 \mathrm{GHz}, 37 \mathrm{GHz}$ and $43 \mathrm{GHz}$ VLBA core long-term light curves from MJD 55750 (2011 July 8) to MJD 56030 (2012 April 13). The flux of the VLBA core at $43 \mathrm{GHz}$ traces the shape of the $37 \mathrm{GHz}$ light curve, indicating that the major part of the total flux originates in there. Moreover, the new components found at $43 \mathrm{GHz}$ are coincident with flux increase in the $37 \mathrm{GHz}$ band. The symbols at the bottom of the plot show the zero separation epochs with the error bars of the components K11 and K12 from the $43 \mathrm{GHz}$ VLBA core (see text).

were performed as a part of the Boston University $\gamma$-ray blazar monitoring programme at $43 \mathrm{GHz}$. The observations were carried out with the VLBA recording system using eight $8 \mathrm{MHz}$ wide channels, each in right and left circular polarization, with 15-20 scans of three to five minutes duration. All ten antennas were used except at epochs affected by weather or receiver failure. The observations are performed about once per month. The data were reduced and modelled in the same manner as described in Jorstad et al. (2005, 2007). In short: the initial correlation was carried out at the National Radio Astronomy Observatory (NRAO) Array Operations Center in Socorro, New Mexico and subsequent calibration was performed with the astronomical image processing system (AIPS) software supplied by NRAO, while images were made with the Caltech software DIFMAP. These calibrations included application of the nominal antenna-based gain curves and system temperatures, as well as correction for sky opacity, followed by iterative imaging plus phase and amplitude self-calibration. The flux-density correction factors from Jorstad et al. (2005) were used for the final adjustment of the flux-density scale in the images. In addition to the kinematics of the jet, the total polarisation data and the polarisation of the VLBA core were also analysed. Also these analysis followed the methods in Jorstad et al. (2005).

\subsection{Results}

In second half of 2011 PKS 1510-089 showed extremely high cm- and mm-band radio flux (Nestoras et al. 2011; Orienti et al. 2011; Beaklini et al. 2011). During the outburst the flux increased from $2 \mathrm{Jy}$ to $7 \mathrm{Jy}$. The outburst peaked first at higher frequencies, the peak at $37 \mathrm{GHz}$ was reached around 2011 October 21 (MJD 55855 ) and at $15 \mathrm{GHz}$ on 2011 December 15 (MJD 55910, see Fig. 8, outburst "11"). After the maximum was reached the two radio light curves showed decreasing flux. However, there are several smaller amplitude outbursts (amplitudes 1-2 Jy) visible in the both light curves peaking at 2012 January 20 and February 25 (MJD 55946 and 55 982) at $15 \mathrm{GHz}$. The last outburst at $15 \mathrm{GHz}$ appears to be a sum of two outbursts seen at $37 \mathrm{GHz}$ peaking at 2012 February 8 and February 25 (MJD 55965 and 55 982, outburst "12a" and "12b" in Fig. 8).

Figure 9 shows radio light curves from all frequencies from the observing campaign period. In the lowest frequencies $(2-8 \mathrm{GHz})$ there is very little variability while at higher frequencies variability is clearly present at all frequencies, but the rather sparse sampling does not allow us to identify outbursts from other than $15 \mathrm{GHz}$ and $37 \mathrm{GHz}$ light curves.

The radio spectral evolution from 2012 January 28 to April 17 (MJD 55954 to 56034) is shown in Fig. 10. In the four first spectra at low frequencies the dominating component is the decaying major outburst. At higher frequencies the new outburst 12a is first visible on 2012 February 18 (MJD 55 975). The outburst 12b is first visible on 2012 March 4 (MJD 55 990) and the peak then moves to lower frequencies. In the last two spectra this outburst is visible as a flattening of the spectra above $15 \mathrm{GHz}$ and increased flux. Both outbursts follow the typical spectral evolution of radio outbursts. In the initial (growth) stage, the synchrotron self-absorption turnover frequency decreases and the turnover flux density increases. In the second (plateau) stage, the turnover frequency decreases while the turnover flux density remains roughly constant. During the third (decay) stage both turnover frequency and flux density decrease. The behaviour is in agreement with the three stage evolution of the shock-in-jet model of Marscher \& Gear (1985); in the first stage the inverse Compton losses dominate, in the second the synchrotron losses and in the third the adiabatic losses.

The VLBA $43 \mathrm{GHz}$ images reveal a new component (named K11) corresponding to the major radio outburst of the second half of 2011 appearing in 2011 December as already reported in Orienti et al. (2013) using the MOJAVE $15 \mathrm{GHz}$ data (see Fig. 11). The apparent speed of the component, $(19.34 \pm 1.85) c$, and the zero separation epoch 2011 October 29 (MJD $55864 \pm 12$ ) agree with ones derived by Orienti et al. (2013). In 2012 April there was a second new component appearing in the images (named K12). It had an apparent speed of $(16.26 \pm 2.43) c$ and a time of ejection of 2012 February 3 (MJD $55961 \pm 15$ ). The zero separation epochs of these 


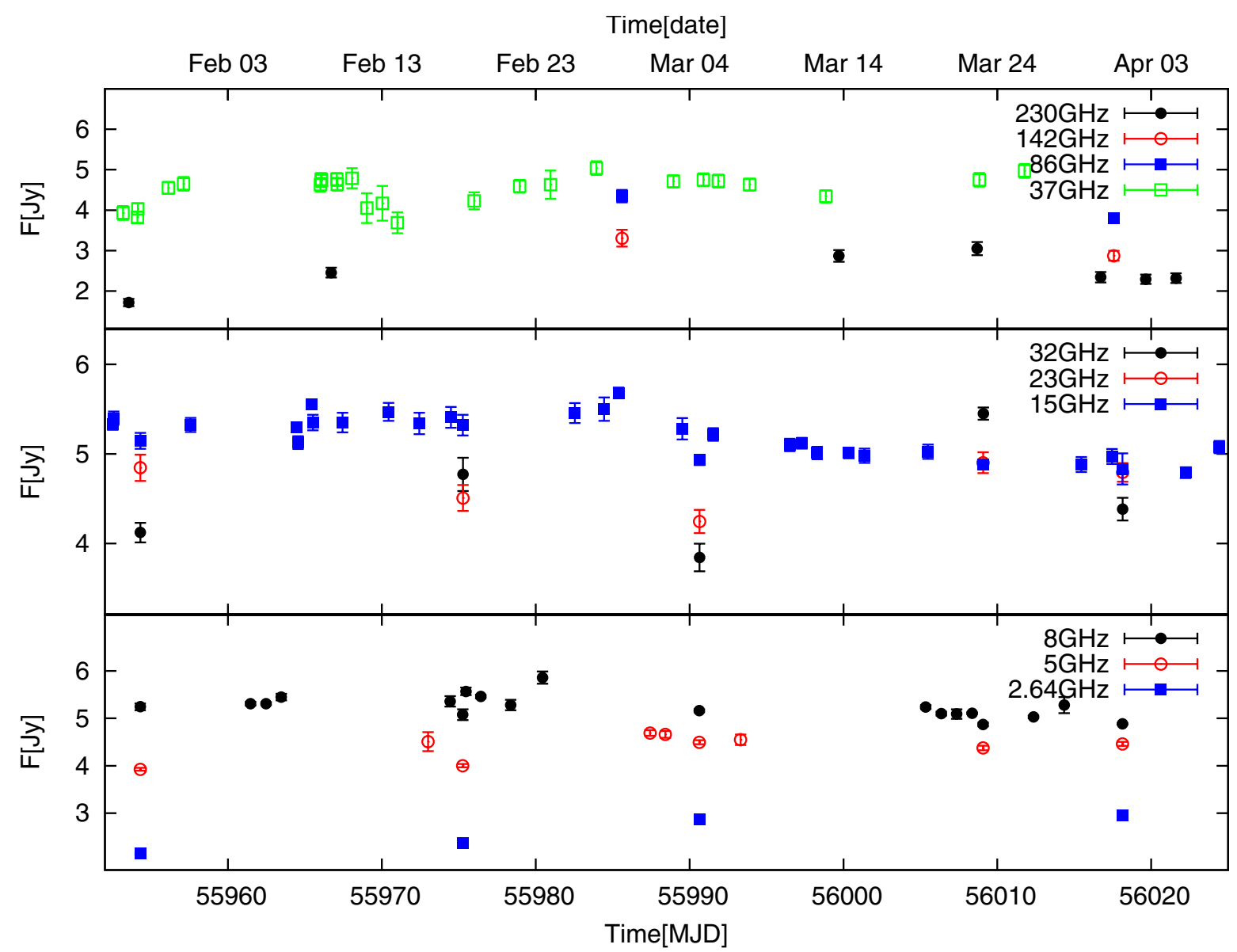

Fig. 9. High frequency (top), medium frequency (middle) and low frequency (bottom) light curves from SMA, Metsähovi, OVRO, UMRAO, Medicina and F-GAMMA programme for the campaign period.

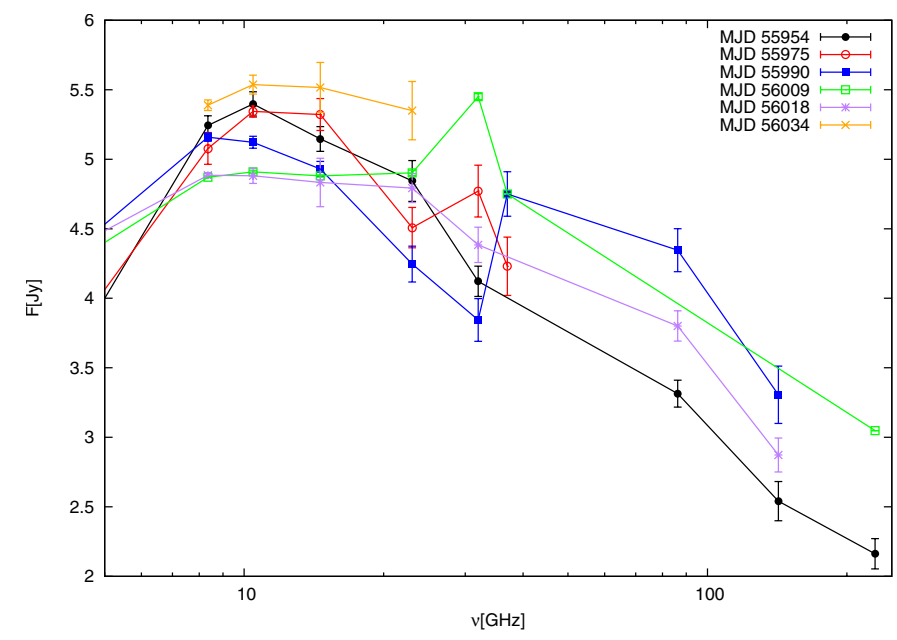

Fig. 10. Evolution of the radio spectra over the campaign period from 2012 January 28 to April 17 (MJD 55 954, 55 975, 55 990, 56 009, 56018 and 56 034). The first radio outburst (11 in Fig. 8) dominates the spectra in the first epoch, while in the second epoch new outburst (12a in Fig. 8) is apparent in the high frequencies. In the third epoch (12b) becomes visible in the highest frequencies with the peak moving to lower energies in the fourth and fifth epochs.

components agree very well with the local maxima in the $37 \mathrm{GHz}$ light curve according to the general trend found in Savolainen et al. (2002). The VLBA polarisation data showed in general a rather low polarisation of the core (1-3\%) compared to the historical values from Jorstad et al. (2007) (0.9-9.7\%). The observed EVPA of the core between 2012 January and April was between $-10^{\circ}$ and $25^{\circ}$. The sparse sampling does not allow us to trace possible rotations of the EVPA, but as shown in Fig. 12, the EVPA values of the VLBA core seem to trace close those of the optical emission.

\section{Multi-frequency light curves}

Figure 13 shows the MAGIC, Fermi-LAT, AGILE-GRID, Swift, optical polarisation, $R$-band photometry and $37 \mathrm{GHz}$ light curves of PKS 1510-089 in 2012 February-April. The Fermi-LAT light curve showed three distinct flares with flux increase more than factor five compared to quiescent state flux: flare I (2012 January 29 to February 13, MJD 55 955-55970), flare II (2012 February 23 to March 9, MJD 55980-55995) and flare III (2012 March 14 to March 19, MJD 56000-56005). Additionally there was a smaller amplitude ( $\sim$ factor four) flare between flare I and II. The first two flares also triggered the AGILE alert system, and are evident in the two day AGILEGRID light curve, while during flare III the source gradually exited the AGILE field of view. As discussed in Sect. 3 AGILE and Fermi-LAT data hint for a marginal harder when brighter trend. During these flares the VHE $\gamma$-ray flux remained rather unchanged. The flares were all characterised by different multifrequency behaviour at lower energies. The first $\gamma$-ray outburst 

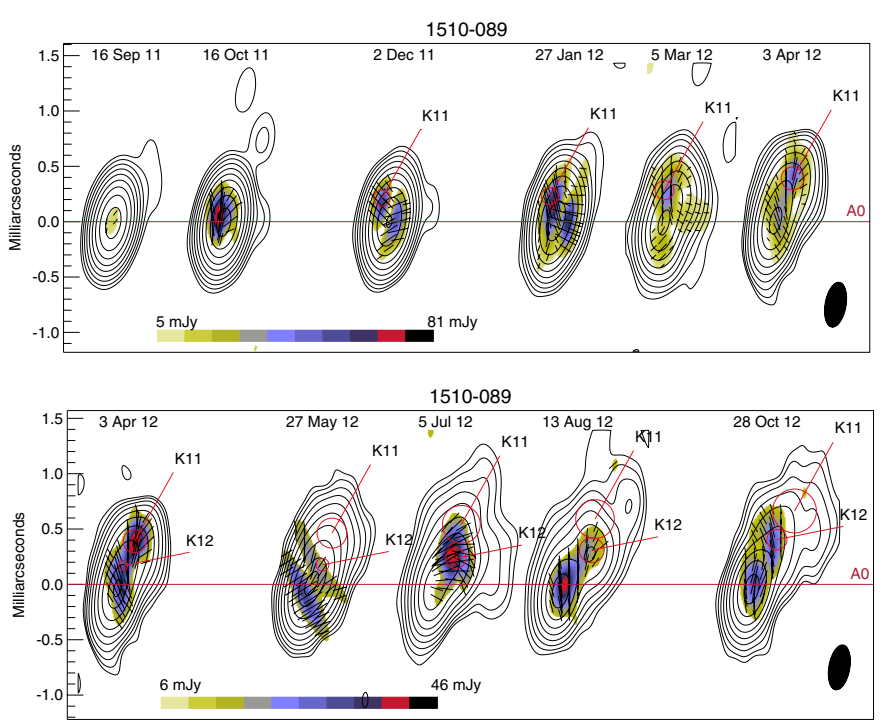

Fig. 11. $43 \mathrm{GHz}$ total (contours) and polarised (colour scale) intensity images of PKS 1510-089 from 2011 September to 2012 April (top) and 2012 April to October (bottom) with $S_{\text {peak }}=2.58 \mathrm{Jy} / \mathrm{beam}$, $S_{\text {peak }}^{\text {pol }}=46 \mathrm{mJy} /$ beam, and a Gaussian restoring beam $=0.14 \times 0.39$ mas $^{2}$ at $\mathrm{PA}=-10^{\circ}$ (in the right bottom corner); contours represent 0.25 , $0.5, \ldots, 64,90 \%$ of the peak intensity; line segments within the colour scale show direction of linear polarisation; solid lines indicate position of components across the epoch, the core A0, knot K11, and knot K12.

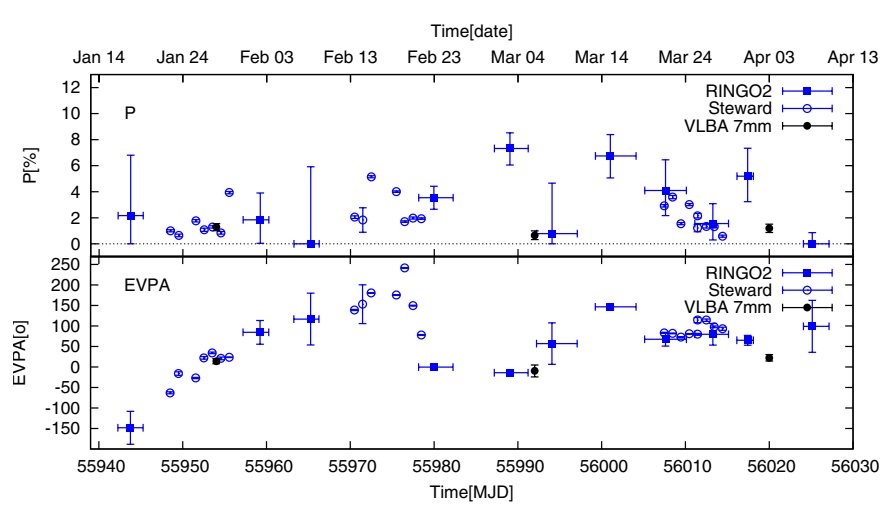

Fig. 12. Radio and optical polarisation behaviour of PKS 1510-089 in 2012 February-April.

coincided with an X-ray peak. The first and second $\gamma$-ray flares were accompanied by quasi simultaneous flares in $37 \mathrm{GHz}$ radio (flare I in $\gamma$ rays is simultaneous to flare $12 \mathrm{a}$ in radio and flare II in $\gamma$ ray is simultaneous to flare $12 \mathrm{~b}$ in radio, see Fig. 13). During the first outburst there was also a rotation of the EVPA of $>180^{\circ}$. This outburst also coincided with the zero separation epoch of new knot from the $43 \mathrm{GHz}$ VLBA core (see Sect. 6.8).

During the second $\gamma$-ray flare there was an optical outburst and in the very beginning a second rotation of the EVPA with $>180^{\circ}$, but this rotation had a very short duration and it was in the opposite direction from the first one. During this rotation there was also a local minimum of the polarisation degree, and this rotation looks very similar to the one observed in 3C 279 during the $\gamma$-ray event seen by Fermi-LAT in 2009 April (Abdo et al. 2010b). However, while the optical flux started to decrease, the $\gamma$-ray flare continued and the optical polarisation degree started to increase.
After these events the EVPA stayed constant until the third rotation started apparently simultaneously with the third outburst in the $\gamma$-ray light curve. During the outburst the degree of polarisation stayed constant. There was a gap in the $37 \mathrm{GHz}$ light curve, however the emission level was similar before and after the gap.

The overall outbursting event had several similarities to the $\gamma$-ray flaring event in 2009 discussed in Marscher et al. (2010); Abdo et al. (2010a); D'Ammando et al. (2011): ejection of the knot from the VLBA core, accompanied activity in the millimetre wavelengths and the rotation of the optical polarisation angle. However, there are also some differences: there was no preceding $\gamma$-ray flare, but the activity in radio and $\gamma$ rays started simultaneously. Also the observed rotation of the optical polarisation angle was shorter in duration ( $\sim 30$ days) and the rotation was only $\sim 380^{\circ}$ instead of $>720^{\circ}$ seen in 2009 .

Marscher et al. (2010) interpreted the 2009 outburst in terms of the phenomenological model presented for BL Lacertae in Marscher et al. (2008). In this model the rotation of the polarisation angle is caused by a moving emission feature following a spiral path as it propagates through the toroidal magnetic field of the acceleration and collimation zones. The emission feature passes the $43 \mathrm{GHz}$ VLBA core, interpreted as a standing conical shock (Marscher et al. 2008), which compresses the knot. The synchrotron flares occur when the energisation of the electrons increases suddenly while the $\gamma$-ray flares with very weak optical counterparts are produced by an increase of the local seed photon field in optical and IR wavelengths. The same scheme can be adopted to the multi-frequency light curve discussed here: flare I takes place as the emission feature passes the core while flare II is caused by the sudden energisation of the electrons of the emission feature and flare III by the sudden increase in the local seed photon field.

Unlike the millimetre, optical and HE $\gamma$ rays, the X-rays did not show strong variability. The $\mathrm{X}$-ray light curve showed a general shape similar to that of the HE $\gamma$-ray light curve. However, the sparse sampling and the small amplitude of variability in the X-ray light curve, do not allow us to draw a strong conclusion on the connection. The X-ray spectrum was hard, as in the previous observations (D'Ammando et al. 2011; Kataoka et al. 2008), which is a signature of a hard electron population with slope 1.6-2.0. The observed properties are in agreement with the conclusion of Kataoka et al. (2008) that the X-ray spectrum is a result of Comptonization of IR radiation produced by hot dust located in the surrounding molecular torus. Direct mid-IR (3.6-160 $\mu \mathrm{m})$ observations give an upper limit on the luminosity from thermal emission from such dust to be $2.3 \times 10^{45} \mathrm{erg} \mathrm{s}^{-1}$ (Malmrose et al. 2011).

As discussed in Sect. 5.7, the behaviour of the optical polarisation EVPA during the observing campaign was particularly interesting showing three distinct rotations of $>180^{\circ}$. In addition to PKS 1510-089 (Marscher et al. 2010) and BL Lac (Marscher et al. 2008) such rotations have been reported for 3C 279 in coincidence with $\gamma$-ray flaring events (Larionov et al. 2008; Abdo et al. 2010b; Aleksić et al. 2011a, 2014). In these papers the rotations have been interpreted as a signature of the geometry, in particular as caused by a bent trajectory that the moving emission feature is following. For 3C 279 the rotations have been changing direction between epochs, which was interpreted as a signature of an actual bend in the jet (Abdo et al. 2010b; Nalewajko et al. 2010). However, here the second rotation was very fast, the rotations took place very close in time and the multi-frequency data suggests that a major part of the emission would originate in 


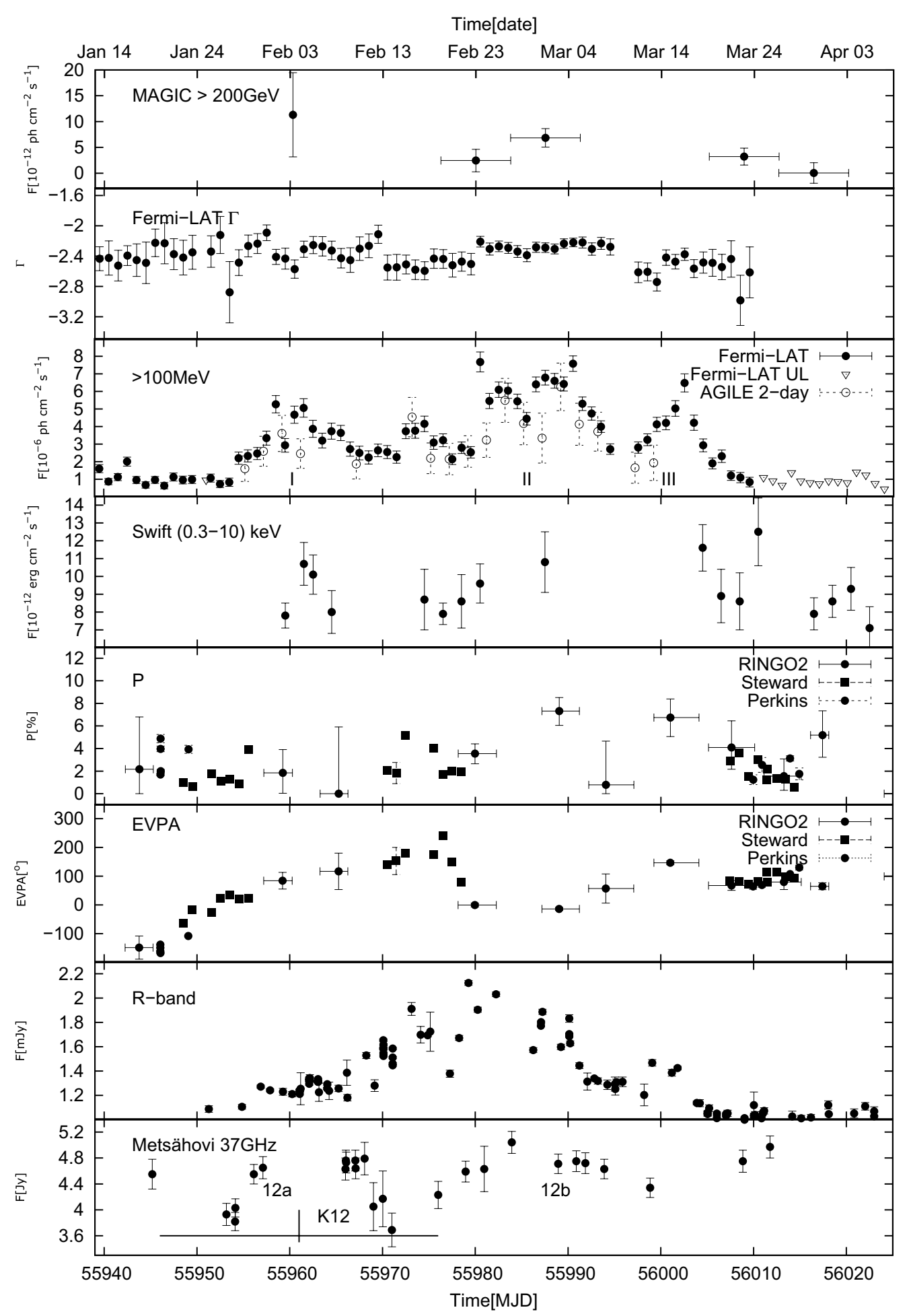

Fig. 13. Multi-frequency light curve of PKS 1510-089 from VHE $\gamma$ rays to radio in February-April 2012. The symbol marked K12 in the bottom panel shows the zero separation epoch of the VLBA component K12 (see Sect. 6.8) from the $43 \mathrm{GHz}$ VLBA core. The numbering of the HE $\gamma$-ray and $37 \mathrm{GHz}$ radio flares is described in the text.

one single emission region. Therefore, a bend does not appear a likely explanation for the change of the direction of the rotations.

As discussed in Marscher et al. (2010, and references therein), the rotations can also be explained by a turbulent magnetic field within the emission region where cells with random magnetic field orientations enter and exit the emission region causing a random walk of the resultant polarisation vector and apparent rotation. For rotations caused by the turbulent magnetic field both directions should be as likely and they should occur at random times. Additionally the appearance of the rotations caused by turbulence is not very smooth. Turbulence as a possible cause of the rotations is favoured by the fact that the rotations with different directions took place very close in time. According to Marscher (2014) such rotations are expected when turbulent plasma flows at relativistic speeds down a jet and crosses the standing shock. 
In summary we conclude that the multi-frequency light curves show compelling evidence that the emission during this flaring epoch is dominated by a moving emission feature located close to the VLBA $43 \mathrm{GHz}$ core. As described before such evidence has been found for PKS 1510-089 as well as in several other sources (e.g. Jorstad et al. 2013, and references therein). The complicated flaring pattern, showing variable synchrotron to Compton ratios and different time scales in diffent wavebands, suggest that additionally both the emission region and the underlying jet might have some substructures.

\section{Spectral energy distribution}

We construct the SED for PKS 1510-089 combining the radio data from F-GAMMA and Metsähovi with infrared, optical and UV data from REM, GASP-WEBT and UVOT, X-ray data from Swift-XRT and $\gamma$-ray data from Fermi-LAT and MAGIC. The radio to X-ray data are quasi-simultaneous taken from 2012 March 1 to March 4 (MJD 55987-55990) while the FermiLAT data cover the main MAGIC observation periods (2012 February 19 to March 5 and March 15 to April 3, MJD 55 97655991 and MJD 56001-56020) and AGILE data the period from 2012 February 20 to March 5 (MJD 55 977.5-55 991.5).

The SEDs of FSRQs are conventionally modelled with a small emission region (typically size of $\sim 10^{16} \mathrm{~cm}$ ) close to the central engine, in regions where the dense radiation field generated by the direct and reprocessed accretion disc emission is thought to provide the ideal environment for efficient IC emission (Dermer \& Schlickeiser 1994; Sikora et al. 1994). There is, however, growing evidence that, at least in some objects and/or at some epochs, the emission could occur far downstream in the jet (Sikora et al. 2008; Marscher et al. 2008; Aleksić et al. 2011b).

For PKS 1510-089 at the epoch analysed here, the multifrequency light curves and the ejection of a new component from the $43 \mathrm{GHz}$ VLBA core point to the co-spatial siting of the $\gamma$-ray and millimetre flaring activity. Since the inner regions of the jet are highly opaque to low frequency photons through synchrotron self-absorption (as indeed observed for the great majority of FSRQs, e.g. Giommi et al. 2012), the $\gamma$-ray and millimetre emission region has to be located farther out in the jet, at distances at which the jet is transparent at radio frequencies. Another compelling indication that the $\gamma$ rays are not produced very close to the nucleus is that, in this case, one would expect a strong depression of the emission above $\sim 20 \mathrm{GeV}$ due to absorption through interactions with the UV-optical emission of the BLR clouds (e.g. Donea \& Protheroe 2003; Sitarek \& Bednarek 2008; Tavecchio \& Mazin 2009; Poutanen \& Stern 2010). Instead, the combined Fermi-LAT and MAGIC $\gamma$-ray spectrum does not show signatures of strong absorption, but a smooth log parabola shape. This is similar to what was observed for PKS 1222+216 (Aleksić et al. 2011b) and for other few other FSRQs whose LAT spectrum extends well above $10-20 \mathrm{GeV}$, supporting the idea of emission occurring beyond the BLR radius (Pacciani et al. 2012; Tavecchio et al. 2013).

The simultaneous millimetre and $\gamma$-ray light curves show similar variability patterns on a weekly time scales, and are therefore consistent with a large dominating emission region, $R \sim c t_{\mathrm{var}} \delta=2 \times 10^{17}(\delta / 10) \mathrm{cm}$, where $\delta$ is the relativistic Doppler factor. The low compactness implied by such large dimensions makes the synchrotron self-Compton process, in which the seed photons are produced in the jet via synchrotron radiation (e.g. Maraschi et al. 1992), highly inefficient. It is thus

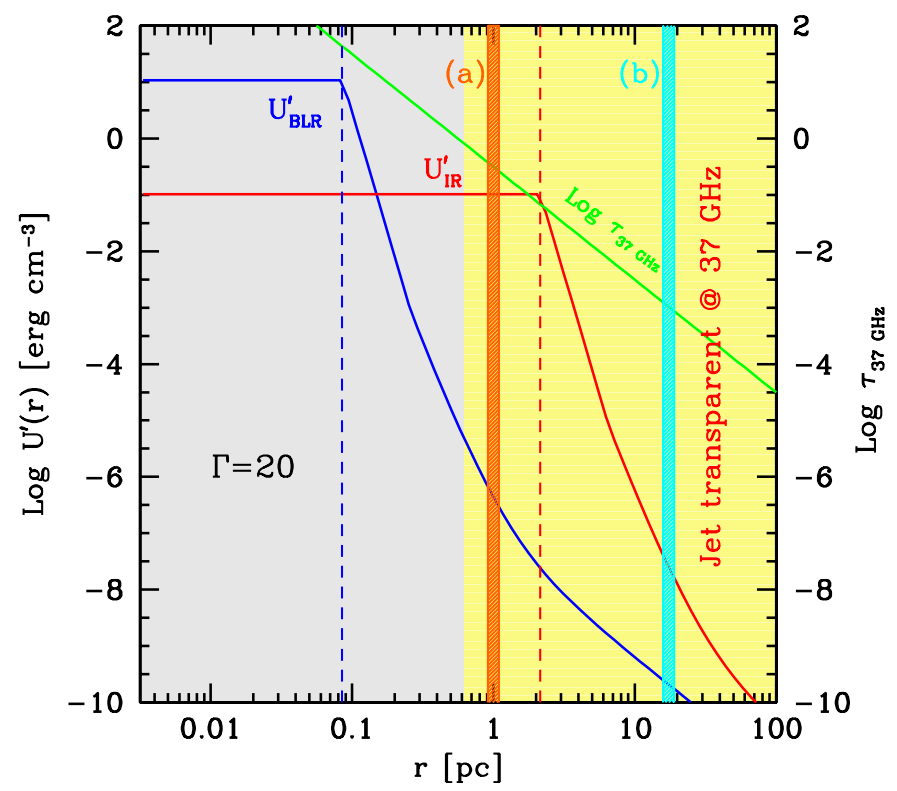

Fig. 14. Energy density of the photon field as function of the distance from the central engine. The blue lines refer to the BLR and the red lines to infrared torus. The green line indicates the $\tau_{37 \mathrm{GHz}}$ (calculated using the magnetic field derived for case $a$ model, see text) and yellow zone indicates the area at which the jet is transparent at $37 \mathrm{GHz}$. The dashed lines indicate the assumed size of BLR (blue) and dust torus (orange). The thick red and cyan vertical lines indicate the regions which we selected for our SED modelling.

unable to produce the observed $\gamma$-ray emission (see e.g. Lindfors et al. 2005, for the case of 3 C 279), and therefore the seed photons for IC scattering must be provided by some external field.

The radiative environment for the jet in PKS 1510-089 is schematically described in Fig. 14, which reports the energy density of the external radiation in the jet co-moving frame as a function of the distance from the central engine. Two components are considered, namely the emission of the BLR clouds in the innermost regions (blue), and the contribution provided by the thermal emission of dust organised in the molecular torus, at larger scales (red). The external energy density is assumed to be constant within the corresponding radius of the emitting structure, $r_{\mathrm{BLR}}$ and $r_{\mathrm{IR}}$, for the BLR and the IR torus respectively, and shows a rapid decline beyond it. The detailed geometry and extension of the BLR and of the IR torus are still under debate, but values typically adopted for the extensions are of order $0.1-1$ parsec and 1-5 parsecs, respectively. Nalewajko et al. (2012) estimated that for PKS 1510-089 these values are $r_{\mathrm{BLR}}=0.07 \mathrm{pc}$ and $r_{\text {Torus }}=3.2 \mathrm{pc}$. The curves in Fig. 14 have been calculated following Ghisellini \& Tavecchio (2009), who provide simple scaling laws for the dimensions of the BLR and the IR torus, depending only on the accretion disc luminosity, $L_{\mathrm{disc}}$. In the literature there are several estimates of the disc luminosity (Celotti et al. 1997; Nalewajko et al. 2012), all in the range $3-7 \times 10^{45} \mathrm{erg} \mathrm{s}^{-1}$. In the following we assume $L_{\text {disc }}=6.7 \times 10^{45} \mathrm{erg} \mathrm{s}^{-1}$, as inferred from the observed "blue bump" traced by UVOT. With the adopted $L_{\text {disc }}$ the estimates of Ghisellini \& Tavecchio (2009) provide $r_{\mathrm{BLR}}=0.086 \mathrm{pc}$ and $r_{\text {Torus }}=2.15 \mathrm{pc}$. The resulting $L_{\text {Torus }}$ is compatible with the upper limits from mid-IR observations (Malmrose et al. 2011).

We reproduced the observed SED by assuming that the emission region (blob) is filled with electrons following a smoothed broken power-law energy distribution with normalization $K$ between $\gamma_{\min }$ and $\gamma_{\max }$, with slopes $n_{1}$ and $n_{2}$ below and above 

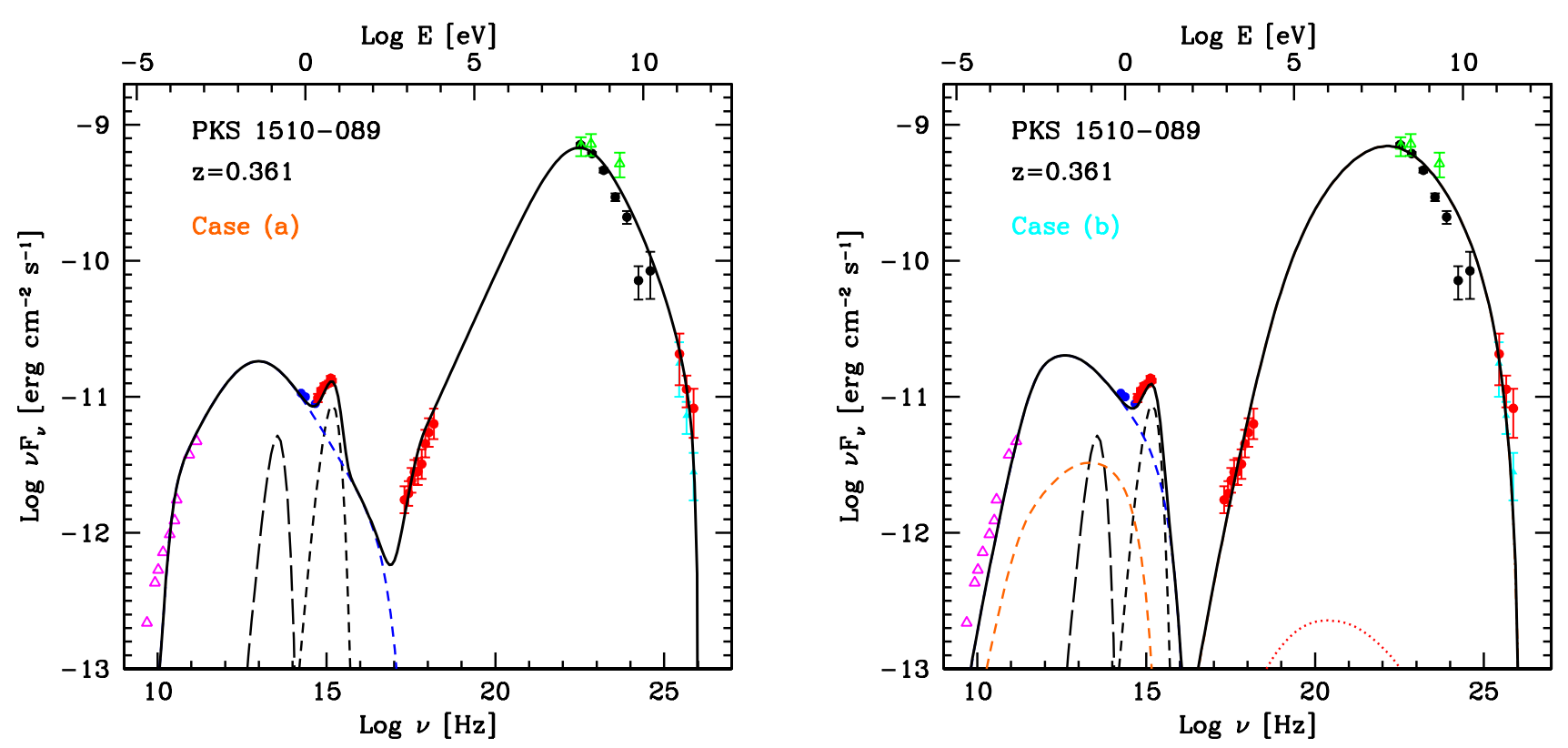

Fig. 15. SED of PKS 1510-089 in 2012 February-April as observed by F-GAMMA and Metsähovi (magenta triangles), GASP-WEBT (blue filled circles), Swift-UVOT and XRT (red filled circles), Fermi-LAT (black filled circles), AGILE-GRID (Flare-II, green triangles) and MAGIC (cyan, observed; red, EBL corrected). Left: the solid black curve shows the overall emission modelled, where the low energy bump is dominated by the synchrotron emission (blue dashed line) and the high energy bump is dominated by the external Compton mechanism, using the infrared torus (long dashed line) photons as seed photons (case $a$ ). The short dashed line is the thermal component from the accretion disc. Right: the black curve shows the model assuming that the emission region is located at the radio core (case $b$ ). The orange dashed line shows the additional external photon field representing the slow sheath of the jet. The red dotted line indicates the synchrotron self Compton emission from this region.

the break at $\gamma_{\mathrm{b}}$ as in Tavecchio et al. (1998) and Maraschi et al. (2003). We assume a conical geometry for the jet, characterised by a semi-aperture angle $\theta_{\mathrm{j}}=0.1^{\circ}$ (Jorstad et al. 2005). Electrons emit through synchrotron and IC mechanisms. The relative luminosities of the IC components arising from the different target photon populations are proportional to the level of the corresponding energy densities (measured in the jet frame), when the scattering takes place in Thomson regime. The energy density, in turn, depends on the distance along the jet as reported in Fig. 14.

The observational evidence discussed above (co-spatiality of $\gamma$-ray and millimetre emission and transparency to $>100 \mathrm{GeV}$ photons) allow us to locate the emission region outside the BLR but do not provide a clear upper limit for its distance. We first tried (case $a$ ) to reproduce the SED finding a solution which minimises the distance from the central engine. The SED is successfully reproduced (Fig. 15) assuming that the emission occurs at a distance of $r \sim 1 \mathrm{pc}$, i.e. within the torus indicated with a thick red vertical line in Fig. 14. As a consistency check, we infer the run of the optical depth at $37 \mathrm{GHz}\left(\tau_{37 \mathrm{GHz}}\right.$, green line in Fig. 14) with the distance (for a conical geometry the scaling laws $B \propto r^{-1}, K \propto r^{-1}$ can be assumed, and adopting, $B$, $K$ and $R$, of the case $a$ model), confirming that the emission region is indeed characterised by $\tau_{37 \mathrm{GHz}}<1$ as required by the correlation observed in the light curves. The grey and the yellow areas in Fig. 14 indicate the opaque and the transparent millimetre regions.

For PKS 1510-089 there are measurements of the distance of the VLBA core from the central engine. Pushkarev et al. (2012) used the core shift measurements to locate the $15 \mathrm{GHz}$ VLBA core at $\sim 17.7$ parsecs from the central engine. Using the speed and core shift measurement from that paper gives a distance of $\sim 6.5$ parsecs (indicated with a thick cyan line in Fig. 14) for the $43 \mathrm{GHz}$ core. At this distance, the infrared torus no longer provides a strong enough source of seed photons for the IC process. Marscher et al. (2010) suggest that the dilemma could be solved if the jet is surrounded by slow sheath providing seed photons for IC scattering. We test this scenario (case $b$ ) by assuming that the emission blob is surrounded by a sheath with $\Gamma=2.2$, so that the radiation field is amplified in the emitting region by a Doppler factor $\delta=3.7$ (assuming a viewing angle $2.8^{\circ}$ and $\Gamma=20$ for the blob). The fit to the SED is presented in Fig. 15: the orange dashed line represents the observed emission from the modelled sheath, which would be negligible compared to the jet emission and therefore not directly observed. We therefore conclude that, from the point of view of the radiative properties, this scenario is also feasible.

We find that both models provide an acceptable fit to the data and the resulting model parameters are given in Table 5. This implies that the presented scenarios are feasible and in agreement with the obtained data; however the parameters used for modelling are not unique. We also note that this SED represents an average emission state, since the data have been collected over a few days, and do not account for the rapid ( $\sim$ one hour) variability of the $\gamma$-ray emission as measured by the Fermi-LAT (Saito et al. 2013; Foschini et al. 2013). As proposed by Marscher et al. (2010; see also Marscher 2014; Narayan \& Piran 2012), such rapid flickering could indicate the presence of relativistic turbulent motion within the flow. In this framework, radiation from single, small, turbulent cells is occasionally observed, while the long-term emission is the result of the integrated emission over all of the active jet volume. More detailed simulations along the lines of Marscher (2014) are required to investigate this scenario in detail.

\section{Summary and discussion}

In this paper we report the detection of VHE $\gamma$ rays from PKS 1510-089 by the MAGIC telescopes in 2012 FebruaryApril. The VHE $\gamma$-ray flux and spectrum are comparable to those 
Table 5. Model parameters for the two SED models.

\begin{tabular}{lccccccccc}
\hline \hline Model & $\gamma_{\min }$ & $\gamma_{\mathrm{b}}$ & $\gamma_{\max }$ & $n_{1}$ & $n_{2}$ & $\begin{array}{c}B \\
{[\mathrm{G}]}\end{array}$ & $\begin{array}{c}K \\
{\left[\mathrm{~cm}^{-3}\right]}\end{array}$ & $\begin{array}{c}R \\
{\left[10^{16} \mathrm{~cm}\right]}\end{array}$ & $\Gamma$ \\
\hline IR torus $^{a}$ & 3 & $9 \mathrm{e} 2$ & $6.5 \mathrm{e} 4$ & 1.9 & 3.85 & 0.12 & 20 & 30 & 20 \\
\hline Sheath $^{b}$ & 800 & $7 \mathrm{e} 3$ & $5 \mathrm{e} 4$ & 2 & 3.4 & $1.3 \mathrm{e}-2$ & 18 & 600 & 2.2 \\
Spine & 800 & $2.6 \mathrm{e} 3$ & $8 \mathrm{e} 4$ & 2 & 3.7 & $6.5 \mathrm{e}-3$ & 2.5 & 510 & 20 \\
\hline
\end{tabular}

Notes. The following quantities are reported: the minimum, break, and maximum Lorentz factors and the low and high energy slope of the electron energy distribution, the magnetic field intensity, the electron density, the radius of the emitting region and the Lorentz factor. ${ }^{(a)}$ IR torus (external photons for IC scattering provided by the IR torus) ${ }^{(b)}$ Sheath-spine (sheath providing the seed photons for the scattering).

observed from the source in 2009 March-April by the H.E.S.S. telescopes (Abramowski et al. 2013). During the MAGIC observations the source was in a high state in the HE $\gamma$-ray band, showing significant variability, but the VHE $\gamma$-ray light curve does not reveal significant variability. This is in agreement with the result of Abramowski et al. (2013).

We performed a detailed multi-frequency study of the source during 2012 January-April extending for the first time from radio to VHE $\gamma$ rays. In summary we find that:

1. The HE and VHE $\gamma$-ray spectra connect smoothly, therefore we conclude that VHE $\gamma$-ray emission and the HE $\gamma$-ray emission originate in a single emission region located outside the BLR.

2. The VHE $\gamma$-ray observations by MAGIC missed the times of the hour scale variability observed in the HE $\gamma$-ray band and the MAGIC light curve does not show significant variability in daily or weekly time scales. However, the HE $\gamma$-ray variability indicates that within the larger emission region, there must exist more compact emission regions producing the fast variability. The model of Marscher (2014), in which turbulent plasma flowing at a relativistic speed down the jet and crossing a standing shock, would naturally lead to such behaviour. We note that, the fast variability could also extend to the VHE $\gamma$-ray band, even if the observations presented here did not detect it.

3. The common variability patterns seen in the HE $\gamma$ ray and $37 \mathrm{GHz}$ light curves as well as the concurrent ejection of a new component from the $43 \mathrm{GHz}$ VLBA core support this emission scenario. We also identify several $\sim 180^{\circ}$ rotations of the optical polarisation angle, which have been suggested as relating to such events (Marscher et al. 2008).

4. The SED can be modelled with a one-zone external Compton model for both studied cases, namely: the seed photons originating from the infrared torus and the seed photons originating from a slow sheath of the jet. The latter model is favoured if the VLBA core is as distant from the central engine as suggested by Marscher et al. (2010); Pushkarev et al. (2012).

However, there are other alternatives for the source of seed photons and for the fast variability:

- Leon-Tavares et al. (2013) suggested that the relativistic jet could drag the broad line region clouds to greater distances from the central engine and the VLBA radio core could be surrounded by such clouds. This would be manifested by a brightening of the broad emission lines in the optical monitoring of the spectral lines. We have no such data for our campaign, but we note that this additional seed photon population is not required to reproduce our data.
- It was proposed in Giannios (2013) that additional flickering can be explained by the jet-in-jet model even if the emission region is far out in the jet. In this scheme long-term flares are the result of the "envelope" emission of magnetic reconnection events in the jet, while short-term flares flag the random formation of "monster" blobs during the reconnection process. While the discussion in Giannios (2013) was suited for the case of PKS $1222+216$, characterised by shorter time scales (both for the long-term modulation and the rapid flares), we expect that it might also be possible to reproduce the behaviour of PKS $1510-089$ by generalising the model.

Since PKS $1510-089$ has been active in $\gamma$ rays in the AGILE and Fermi-LAT era, there have been several other multi-frequency studies. Brown et al. (2013) analysed the Fermi-LAT data concluding from the presence of $>20 \mathrm{GeV}$ photons that multiple simultaneously active $\gamma$-ray emission regions are required. We find that the Fermi-LAT and MAGIC spectra connect smoothly suggesting a single emission region. These very fast spikes probably originate in a separate emission region, possibly embedded in the larger region producing the slower modulations of the radio and $\gamma$-ray light curves. Several emission sites were also suggested by Nalewajko et al. (2012), who concluded that the high energy cut-off (in the low state) of the main synchrotron component implies a two-zone model, otherwise the requested external photon density is too high. Barnacka et al. (2014) reached a similar conclusion and favoured a two-zone model for reproducing the VHE $\gamma$-ray emission observed by H.E.S.S. telescopes. However, in our modelling, one emission region is sufficient to reproduce the average SED during the high state.

In addition to PKS 1510-089, only two other FSRQs have been detected in VHE $\gamma$ rays (3C 279 and PKS 1222+216). All detections have been made during a high state in the lower energy regimes and even during high activity in the HE $\gamma$-ray band, 3C 279 (Aleksić et al. 2014) and PKS 1222+216 (Ackermann et al. 2014) are detected in VHE $\gamma$ rays only during individual nights. The upper limits derived for these two sources from the non-detections are also below the detected flux. In this sense PKS 1510-089 is clearly different from the other two and follow up observations in lower HE $\gamma$-ray states should be performed in order to study if the source is a constant VHE $\gamma$-ray emitter like some of the high peaking BL Lac objects detected in VHE $\gamma$ rays (e.g. Aliu et al. 2012). However, VHE $\gamma$-ray detections from FSRQs imply that in all cases the emission takes place outside the BLR (Aleksić et al. 2011b,a). Further observations are needed to study why in some cases we see extremely bright, fast flares of VHE $\gamma$ rays and in other cases (as in the case of PKS 1510-089) the emission of VHE $\gamma$ rays appears more stable. 
Acknowledgements. We would like to thank the Instituto de Astrofísica de Canarias for the excellent working conditions at the Observatorio del Roque de los Muchachos in La Palma. The support of the German BMBF and MPG, the Italian INFN, the Swiss National Fund SNF, and the Spanish MICINN is gratefully acknowledged. This work was also supported by the CPAN CSD2007-00042 and MultiDark CSD2009-00064 projects of the Spanish Consolider-Ingenio 2010 programme, by grant DO02-353 of the Bulgarian NSF, by grant 127740 of the Academy of Finland, by the DFG Cluster of Excellence "Origin and Structure of the Universe", by the DFG Collaborative Research Centres SFB823/C4 and SFB876/C3, by the Polish MNiSzW grant 745/N-HESS-MAGIC/2010/0, the University of Rijeka Project 13.12.1.3.02, the Croatian Science Foundation Project 09/176 and by JSPS KAKENHI Grants numbers 24000004. J. S. is supported by ERDF and the Spanish MINECO through FPA2012-39502 and JCI-2011-10019 grants. K. S. is supported by JSPS KAKENHI grant number 25800105. The Fermi-LAT Collaboration acknowledges generous ongoing support from a number of agencies and institutes that have supported both the development and the operation of the LAT as well as scientific data analysis. These include the National Aeronautics and Space Administration and the Department of Energy in the United States, the Commissariat à l'Énergie Atomique and the Centre National de la Recherche Scientifique/Institut National de Physique Nucléaire et de Physique des Particules in France, the Agenzia Spaziale Italiana and the Istituto Nazionale di Fisica Nucleare in Italy, the Ministry of Education, Culture, Sports, Science and Technology (MEXT), High Energy Accelerator Research Organization (KEK) and Japan Aerospace Exploration Agency (JAXA) in Japan, and the K. A. Wallenberg Foundation, the Swedish Research Council and the Swedish National Space Board in Sweden. Additional support for science analysis during the operations phase is gratefully acknowledged from the Istituto Nazionale di Astrofisica in Italy and the Centre National d'Études Spatiales in France. Astrorivelatore Gamma ad Immagini LEggero (AGILE) is a scientific mission of the Italian Space Agency (ASI) with INFN, INAF and CIFS participation. AGILE research partially supported through the ASI grants I/089/06/2, $\mathrm{I} / 042 / 10 / 0$ and I/028/12/0. Data from the Steward Observatory spectropolarimetric monitoring project were used. This programme is supported by Fermi Guest Investigator grants NNX08AW56G, NNX09AU10G, and NNX12AO93G. This article is partly based on observations made with the telescopes IAC80 and TCS operated by the Instituto de Astrofisica de Canarias in the Spanish Observatorio del Teide on the island of Tenerife. Most of the observations were taken under the rutinary observation programme. The IAC team acknowledges the support from the group of support astronomers and telescope operators of the Observatorio del Teide. The Abastumani team acknowledges financial support of the project FR/638/6-320/12 by the Shota Rustaveli National Science Foundation under contract $31 / 77$. The OVRO $40-\mathrm{m}$ monitoring programme is supported in part by NASA grants NNX08AW31G and NNX11A043G, and NSF grants AST-0808050 and AST-1109911. The VLBA is operated by the National Radio Astronomy Observatory. The National Radio Astronomy Observatory is a facility of the National Science Foundation operated under cooperative agreement by Associated Universities, Inc. The research at Boston University (BU) was funded in part by NASA Fermi Guest Investigator grants NNX11AQ03G, NNX11AO37G, and NNX12AO90G. The PRISM camera at Lowell Observatory was developed by K. Janes et al. at BU and Lowell Observatory, with funding from the NSF, BU, and Lowell Observatory. The Submillimeter Array is a joint project between the Smithsonian Astrophysical Observatory and the Academia Sinica Institute of Astronomy and Astrophysics and is funded by the Smithsonian Institution and the Academia Sinica. The Metsähovi team acknowledges the support from the Academy of Finland to our observing projects (numbers 212656, 210338, 121148, and others). This research is partly based on observations with the $100 \mathrm{~m}$ telescope of the MPIfR (Max-Planck-Institut für Radioastronomie) at Effelsberg and with the IRAM 30-m telescope. IRAM is supported by INSU/CNRS (France), MPG (Germany) and IGN (Spain). I. Nestoras is funded by the International Max Planck Research School (IMPRS) for Astronomy and Astrophysics at the Universities of Bonn and Cologne.

\section{References}

Abdo, A. A., Ackermann, M., Agudo, I., et al. (Fermi-LAT Collaboration) 2010a, ApJ, 721, 1425

Abdo, A. A., Ackermann, M., Ajello, M., et al. (Fermi-LAT Collaboration) 2010b, Nature, 463, 919

Abramowski, A., Acero, F., Aharonian, F., et al. (H.E.S.S. Collaboration) 2013, A\&A, 554, 107

Ackermann, M., Ajello, M., Allafort, A., et al. (Fermi-LAT Collaboration) 2012, ApJ, 747, 104

Ackermann, M., Ajello, M., Allafort, A., et al. (Fermi-LAT and MAGIC Collaborations) 2014, ApJ, 786, 157

Albert, J., Aliu, E., Anderhub, H., et al. (MAGIC Collaboration) 2007, ApJ, 669, 862
Albert, J., Aliu, E., Anderhub, H., et al. (MAGIC Collaboration) 2008a, Science, 320,1752

Albert, J., Aliu, E., Anderhub, H., et al. (MAGIC Collaboration) 2008b, Nucl. Instr. Meth. A, 588, 424

Aleksić, J., Anderhub, H., Antonelli, L. A., et al. (MAGIC Collaboration) 2010, A\&A, 519, 32

Aleksić, J., Anderhub, H., Antonelli, L. A., et al. (MAGIC Collaboration) 2011a, A\&A, 530, A4

Aleksić, J., Anderhub, H., Antonelli, L. A., et al. (MAGIC Collaboration) 2011b, ApJ, 730, L8

Aleksić, J., Alvarez, E. A., Antonelli, L. A., et al. (MAGIC Collaboration) 2012, Astropart. Phys, 35, 435

Aleksić, J., Antonelli, L. A., Antoranz, P., et al. (MAGIC Collaboration) 2013, A\&A, 556, A67

Aleksić, J., Ansoldi, S., Antonelli, L. A., et al. (MAGIC Collaboration) 2014, A\&A, accepted

Aliu, E., Anderhub, H., Antonelli, L. A., et al. (MAGIC Collaboration) 2009, ApJ, 692, L29

Aliu, E., Archambault, S., Arlen, T., et al. (VERITAS Collaboration) 2012, ApJ, 755,118

Angelakis, E., Fuhrmann, L., Marchili, N., Krichbaum, T. P., \& Zensus, J. A. 2008, Mem. Soc. Astron. It., 79, 1042

Arnaud, K. A. 1996, ASP Conf. Ser., 101, 17

Atwood, W. B., Abdo, A. A., Ackermann, M., et al. 2009, ApJ, 697, 1071

Baars, J. W. M., Genzel, R., Pauliny-Toth, I. I. K., \& Witzel, A. 1977, A\&A, 61, 1,99

Barnacka, A., Moderski, R., Behera, B., Brun, P., \& Wagner, S. 2014, A\&A, 567, A113

Barthelmy, S. D., Barbier, L. M., Cummings, J. R., et al. 2005, SSRv, 120, 143

Beaklini, P., Abraham, Z., \& Dominici, T. 2011, ATel, 3799

Bertero, M. 1989, Advances in Electronics and Electron Physics (New York: Academic Press Inc.), 75

Brown, A. M. 2013, MNRAS, 431, 824

Bulgarelli, A., Chen, A. W., Tavani, M., et al. 2012 A\&A, 540, A79

Burrows, D. N., Hill, J. E., Nousek, J. A., et al. 2005, Space Sci. Rev., 120, 165

Böttcher M., Reimer, A., \& Marscher, A. P. 2009, ApJ, 703, 1168

Cardelli, J. A., Clayton, G. C., \& Mathis, J. S. 1989, ApJ, 345, 245

Cash, W. 1979, ApJ, 228, 939

Celotti, A., Padovani, P., \& Ghisellini, G. 1997, MNRAS, 286, 415

Chatterjee, R., Jorstad, S. G., Marscher, A. P., et al. 2008, ApJ, 689, 79

Chincarini, G., Zerbi, F. M., Antonelli, L. A., et al. 2003, The Messenger, 113, 40

Cortina, J. (on behalf of the MAGIC Collaboration) 2012, ATel, 3975

Covino, S., Zerbi, F. M., Chincarini, G., et al. 2004a, Astron Nachr., 325, 543

Covino, S., Stefanon, M., Sciuto, G., et al. 2004b, SPIE, 5492, 1613

D’Ammando, F., Vercellone, S., Tavani, M., et al. 2009, ATel, 1957

D'Ammando, F., Raiteri, C. M., Villata, M., et al. 2011, A\&A, 529, A145

De Caneva, G., Barres de Almeida, U., Becerra Gonzelez, J. et al. (on behalf of the MAGIC Collaboration) 2012, Proc. 5th Int. Symp. on High-Energy Gamma-Ray Astronomy, AIP Conf. Proc. 1505, 502

Devillard, N. 1997, The Messenger, 87, 19

Dermer, C., \& Schlickeiser, R., 1994, ApJS, 90, 945

Dominguez, A., Primack, J. R., Rosario, D. J., et al. 2011, MNRAS, 410, 2556

Donea, A.-C., \& Protheroe, R. J. 2003, Astropart. Phys., 18, 377

Donnarumma, I., Lucarelli, F., Vercellone, S., et al. 2011, ATel, 3470

Fomin, V. P., Stepanian, A. A., Lamb, R. C., et al. 1994, Astropart. Phys., 2, 137

Foschini, L., Bonnoli, G., Ghisellini, G., et al. 2013, A\&A, 555, A138

Franceschini, A., Rodighiero, G., \& Vaccari, M. 2008, A\&A, 487, 837

Fuhrmann, L., Zensus, J. A., Krichbaum, T. P., Angelakis, E., \& Readhead,

A. C. S. 2007, The First GLAST Symp., 921, 249

Fuhrmann, L., Krichbaum, T. P., Witzel, A., et al. 2008, A\&A, 490, 1019

Gehrels, N., Chincarini, G., Giommi, P., et al. 2004, ApJ, 611, 1005

Ghisellini, G., Celotti, A., Fossati, G., et al. 1998, MNRAS, 301, 451

Giannios, D. 2013, MNRAS, 431, 355

Giommi, P., Polenta, G., Lähteenmäki, A., et al. 2012, A\&A, 541, A160

Giuliani, A., Chen, A., Mereghetti, S., et al. 2004, Mem. Soc. Astron. It. Suppl., 5,135

Gurwell, M. A., Peck, A. B., Hostler, S. R., et al. 2007, ASP Conf. Ser., 375, 234

Górski, K. M., Hivon, E., Banday, A. J., et al. 2005, ApJ, 622, 759

Hartman, R. C., Bertsch, D. L., Bloom, S. D., et al. 1999, ApJS, 123, 79

Hartman, R. C., Böttcher, M., Aldering, G., et al. 2001, ApJ, 553, 683

Hauser, M. G., \& Dwek, E. 2001, ARA\&A, 39, 249

Jorstad, S. G., Marscher, A. P., Mattox, J. R., et al. 2001, ApJ, 556, 738 
Jorstad, S. G., Marscher, A. P., Lister, M. L., et al. 2005, AJ, 130, 1418 Jorstad, S. G., Marscher, A. P., Stevens, J. A., et al. 2007, AJ, 134, 799 Jorstad, S. G., Marscher, A. P., Smith, P. S., et al. 2011, BAAS, 217, 40804 Jorstad, S. G., Marscher, A. P., et al. 2013, ApJ, 773, 147

Kalberla, P. M. W., Burton, W. B., Hartmann, D., et al. 2005, A\&A, 440, 775 Kaspi, S., Smith, P. S., Netzer, H., et al. 2000, ApJ, 533, 631

Kataoka, J., Madejski, G., Sikora, M., et al. 2008, ApJ, 672, 787

Krimm, H. A., Holland, S. T., Corbet, R. H. D., et al. 2013, ApJS, 209, 14

Larionov, V., Jorstad, S. G., Marscher, A. P., et al. 2008, A\&A, 492, 389

Leon-Tavares, J., Chavushyan, V., Patino-Alvarez, V., et al. 2013, ApJ, 763, 36

Li, T. P., \& Ma, Y. Q. 1983, ApJ, 272, 317

Lindfors, E., Valtaoja, E., \& Türler, M. 2005, A\&A, 440, 845

Lindfors, E., Türler, M., Valtaoja, E., et al. 2006, A\&A, 456, 895

Lindfors, E., Nilsson, K., Barres de Almeida, U., et al. (on behalf of the MAGIC Collaboration), 2012, Fermi Symp. Proc. - eConf C121028 [arXiv: 1303.2102]

Lucarelli, F., Piano, G., Verrecchia, F., et al. 2012, ATel, 3934

Lähteenmäki, A., \& Valtaoja, E., 2003, ApJ, 590, 92

Malmrose, M. P., Marscher, A. P., Jorstad, S. G., Nikutta, R., \& Elitzur, M. 2011, ApJ, 732, 116

Maraschi, L., \& Tavecchio, F. 2003, ApJ, 593, 667

Maraschi, L., Ghisellini, G., \& Celotti, A. 1992, ApJ, 397, 5

Marscher, A. 2014, ApJ, 780, 87

Marscher, A. P., \& Gear, W. K., 1985, ApJ, 298, 114

Marscher, A., Jorstad, S., D'Arcangelo, F. D., et al. 2008, Nature, 452, 966

Marscher, A., Jorstad, S., Larionov, V., et al. 2010, ApJ, 710, L126

Marscher, A., Jorstad, S., Agudo, I., et al. 2012, Fermi \& Jansky Proc. - eConf C1111101 [arXiv: 1204.6707]

Mattox, J. R., Bertsch, D. L., Chiang, J., et al. 1996, ApJ, 461, 396

Moralejo, A., Gaug, M., Carmona, E., et al. 2009, in Proc. 31st ICRC (Łódź), 469 [arXiv:0907.0943]

Naghizadeh-Khouei, J., \& Clarke, D. 1993, A\&A, 274, 968

Nalewajko, K. 2010, Int. J. Mod. Phys. D, 19, 701

Nalewajko, K., Sikora, M., Madejeski, G., et al. 2012, ApJ, 760, 69

Narayan, R., \& Piran, T. 2012, MNRAS, 420, 1, 604

Nolan, P. L., Abdo, A. A., Ackermann, M., et al. 2012, ApJS, 199, 31

Nestoras, I., Fuhrmann, L., Angelakis, E., et al. 2011, ATel, 3698

Orienti, M., D'Ammando, F., Giroletti M., et al. 2011, ATel, 3775

Orienti, M., D'Ammando, F., Giroletti, M., et al. 2013, MNRAS, 428, 241

Pacciani, L., Donnarumma, I., Denney, K. D., et al. 2012, MNRAS, 425, 2015

Pittori, C., Verrecchia, F., Chen, A. W., et al. 2009 A\&A, 506, 1563

Poole, T. S., Breeveld, A. A., Page, M. J., et al. 2008, MNRAS, 383, 62

Poutanen, J., \& Stern, B. 2010, ApJ, 717, L118

Pucella, G., Vittorini, V., D’Ammando F., et al. 2008, A\&A, 491, L21

Pushkarev, A. B., Hovatta, T., Kovalev, Y. Y., et al. 2012, A\&A, 545, 113

Raiteri, C. M., Villata, M., de Francesco, G., et al. 1998, A\&AS, 130, 495

Raiteri, C. M., Villata, M., Larionov, V. M., et al. 2008, A\&A, 491, 755

Raiteri, C. M., Villata, M., Bruschini, L., et al. 2010, A\&A, 524, A43

Raiteri, C. M., Villata, M. Smith, P. S., et al. 2012, A\&A, 545, A48

Roming, P. W. A., Kennedy, T. E., Mason, K. O., et al. 2005, Space Sci. Rev., 120,95

Richards, J. L., Max-Moerbeck, W., Pavlidou, V., et al. 2011, ApJS, 194, 29

Saito, S., Stawarz, L., Tanaka, Y., et al. 2013, ApJ, 766, 11

Savolainen, T., Wiik, K., Valtaoja, E., et al. 2002, A\&A, 394, 851

Schlegel, D. J., Finkbeiner, D. P., Davis, M., et al. 1998, ApJ, 500, 525

Sikora, M., Begelman, M. C., \& Rees, M. J. 1994, ApJ, 421, 153

Sikora, M., Moderski, R., \& Madejski, G. M. 2008, ApJ, 675, 71

Sikora, M., Stawarz, L., Moderski, R. Nalewajko, K., \& Madejski, G. M. 2009, ApJ, 704, 38

Sitarek, J., \& Bednarek, W. 2008, MNRAS, 391, 624

Sitarek, J., Gaug, M., Mazin, D., Paoletti, R., \& Tescaro, D. 2013, Nucl. Inst. Meth. Phys. Res. A, 723, 109

Smith, P. S., Montiel, E., Rightley, S., et al. 2009, Fermi Symp., eConf Proc. C091122 [arXiv: 0912.3621]

Stecker, F. W., de Jager, O. C., \& Salamon, M. H. 1992, ApJ, 390, L49

Striani, E., Verrecchia F., Tavani M., et al. 2010, ATel, 2385

Tavani, M., Barbiellini, G., Argan, A., et al. 2009, A\&A, 502, 995

Tavecchio, F., \& Mazin, D. 2009, MNRAS, 392, 40

Tavecchio, F., Maraschi, L., \& Ghisellini, G. 1998, ApJ, 509, 608

Tavecchio, F., Pacciani, L., Donnarumma, I., et al. 2013, MNRAS, 435,24

Teraesranta, H., Tornikoski, M., Mujunen, A., et al. 1998, A\&AS, 132, 305

Vercellone, S., D’Ammando, F., Vittorini, V., et al. 2010, ApJ, 712, 405

Verrecchia, F., Pittori, C., Cardillo, M., et al. 2012, ATel, 3907

Verrecchia, F., Pittori, C., Chen, A., et al. 2013, A\&A, 558, A137

Villata, M., Raiteri, C. M., Ghisellini, G., et al. 1997, A\&AS, 121, 119

Wagner, S. (H.E.S.S. Collaboration) 2010, HEAD meeting \#11, \#7.05; BAAS, 42,660
Zerbi, F. M., Chincarini, G., Ghisellini, G., et al. 2001, Astron. Nachr., 322, 275

1 IFAE, Campus UAB, 08193 Bellaterra, Spain

2 Università di Udine, and INFN Trieste, 33100 Udine, Italy

3 INAF National Institute for Astrophysics, 00136 Rome, Italy

4 Università di Siena, and INFN Pisa, 53100 Siena, Italy

5 Croatian MAGIC Consortium, Rudjer Boskovic Institute, University of Rijeka and University of Split, 10000 Zagreb, Croatia

6 Max-Planck-Institut für Physik, 80805 München, Germany

7 Universidad Complutense, 28040 Madrid, Spain

8 Inst. de Astrofísica de Canarias, 38200 La Laguna, Tenerife, Spain

9 University of Łódź, 90236 Lodz, Poland

10 Deutsches Elektronen-Synchrotron (DESY), 15738 Zeuthen, Germany

11 ETH Zurich, 8093 Zurich, Switzerland

12 Universität Würzburg, 97074 Würzburg, Germany

13 Centro de Investigaciones Energéticas, Medioambientales y Tecnológicas, 28040 Madrid, Spain

14 Technische Universität Dortmund, 44221 Dortmund, Germany

15 Inst. de Astrofísica de Andalucía (CSIC), 18080 Granada, Spain

16 Università di Padova and INFN, 35131 Padova, Italy

17 Università dell'Insubria, Como, 22100 Como, Italy

18 Unitat de Física de les Radiacions, Departament de Física, and CERES-IEEC, Universitat Autònoma de Barcelona, 08193 Bellaterra, Spain

19 Institut de Ciències de l'Espai (IEEC-CSIC), 08193 Bellaterra, Spain

20 Japanese MAGIC Consortium, Division of Physics and Astronomy, Kyoto University, 606-8901 Kyoto, Japan

21 Finnish MAGIC Consortium, Tuorla Observatory, University of Turku and Department of Physics, University of Oulu, 900147 Oulu, Finland

22 Inst. for Nucl. Research and Nucl. Energy, 1784 Sofia, Bulgaria

23 Universitat de Barcelona, ICC, IEEC-UB, 08028 Barcelona, Spain

24 Università di Pisa, and INFN Pisa, 56126 Pisa, Italy

25 now at NASA Goddard Space Flight Center, Greenbelt MD 20771; and Department of Physics and Department of Astronomy, University of Maryland, College Park, MD 20742, USA

26 now at Ecole polytechnique fédérale de Lausanne (EPFL), 105 Lausanne, Switzerland

27 now at Department of Physics \& Astronomy, UC Riverside CA 92521, USA

28 now at Finnish Centre for Astronomy with ESO (FINCA), 21500 Piikkiö, Turku, Finland

29 also at INAF-Trieste, 34131 Trieste, Italy

30 also at Instituto de Fisica Teorica, UAM/CSIC, 28049 Madrid, Spain

31 now at Stockholm University, Oskar Klein Centre for Cosmoparticle Physics, 10691 Stockholm, Sweden

32 now at GRAPPA Institute, University of Amsterdam, 1098XH Amsterdam, The Netherlands

33 Dipartimento di Fisica, Università degli Studi di Perugia, via A. Pascoli, 06123 Perugia; INFN Sezione di Perugia, via A. Pascoli, 06123 Perugia, Italy

34 ASI Science Data Centre (ASDC), via del Politecnico snc, 00133 Roma, INAF-OAR, via Frascati 33, 00040 Monte Porzio Catone (RM), Italy

35 INAF-IASF Palermo, via Ugo La Malfa 153, 90146 Palermo, Italy

36 Astrophysics Research Institute, Liverpool John Moores University, Twelve Quays House, Egerton Wharf, Birkenhead, CH41 1LD, UK

37 Tuorla Observatory, Department of Physics and Astronomy, University of Turku, 21500 Piikkiö, Finland

38 INAF - Osservatorio Astrofisico di Torino, 10025 Pino Torinese Torino, Italy

39 Aalto University Metsähovi Radio Observatory, Metsähovintie 114, 02540 Kylmälä, Finland

40 Aalto University Department of Radio Science and Engineering, 02150 Espoo, Finland 
41 Cahill Center for Astronomy \& Astrophysics, Caltech, 1200 E. California Blvd, Pasadena CA 91125, USA

42 Department of Physics, Purdue University, 525 Northwestern Ave, West Lafayette IN 47907, USA

43 Institute for Astrophysical Research, Boston University, USA

44 INAF-IRA Bologna, Italy

45 Harvard-Smithsonian Center for Astrophysics, Cambridge MA 02138, USA

46 Astron. Inst., St.-Petersburg State Univ., Russia

47 Pulkovo Observatory, St.-Petersburg, Russia

48 Isaac Newton Institute of Chile, St.-Petersburg Branch, Russia

49 University of Crete, Heraklion, Greece

50 Graduate Institute of Astronomy, National Central University, 300 Jhongda Rd., Jhongli 32001, Taiwan

51 Joint Institute for VLBI in Europe, Postbus 2, 7990 AA Dwingeloo, The Netherlands

52 Instituto de Astrofísica de Andalucía, CSIC, Apartado 3004, 18080 Granada, Spain

53 Abastumani Observatory, Mt. Kanobili, 0301 Abastumani, Georgia
54 Landessternwarte, Zentrum für Astronomie der Universität Heidelberg, Königstuhl 12, 69117 Heidelberg, Germany

55 Engelhardt Astronomical Observatory, Kazan Federal University, 422526 Tatarstan, Russia

56 Instituto de Astrofisica de Canarias (IAC), 38205 San Critóbal, La Laguna, Tenerife, Spain

57 Departamento de Astrofisica, Universidad de La Laguna, 38206 La Laguna, Tenerife, Spain

58 Sofia University, 1000 Sofia, Bulgaria

59 Department of Astronomy, University of Michigan, 817 Dennison Bldg., Ann Arbor MI 48109-1042, USA

${ }^{60}$ Max-Planck-Institut für Radioastronomie, Auf dem Huegel 69, 53121 Bonn, Germany

61 Institut de Radio Astronomie Millimétrique, Avenida Divina Pastora 7, Local 20, 18012 Granada, Spain

62 Institute of Space and Astronautical Science, JAXA, 3-1-1 Yoshinodai, Chuo-ku, Sagamihara, 252-5210 Kanagawa, Japan

63 Astronomical Observatory, Jagiellonian University, 30-244 Krakow, Poland 